\title{
Covariance-map imaging: a powerful tool for chemical dynamics studies
}

\author{
Claire Vallance*, David Heathcote, and Jason W. L. Lee \\ Department of Chemistry, University of Oxford, Chemistry Research \\ Laboratory, 12 Mansfield Rd, Oxford OX1 3TA, UK \\ *e-mail: claire.vallance@chem.ox.ac.uk
}

\begin{abstract}
Over the past decade or so, the state-of-the-art in the field of chemical reaction dynamics has progressed from studies of few-atom systems to wide-ranging investigations into a variety of photoinduced and collision-induced processes in much larger molecules. Many of these studies are of direct relevance to a wide audience of chemists, spanning fields such as atmospheric chemistry, astrochemistry, synthetic chemistry, and chemical biology. Key to this work has been the technique of velocity-map imaging, which allows complete product scattering distributions to be recorded for the process of interest. Recent advances in camera technology have enabled the development of multi-mass velocity-map imaging, in which the scattering distributions of all reaction products can be recorded in a single measurement. In addition to the scattering distributions of individual reaction products, the data set now contains information on correlations between the scattering distributions of two or more fragments. These correlations can be revealed using the technique of statistical covariance, yielding an approach known as covariance-map imaging. This review will introduce the reader to covariance mapping, and will describe various applications of the technique within the field of chemical dynamics. The underlying concepts will be illustrated through a series of simple simulations, before moving on to describe a number of recent experimental studies in which covariance mapping has been used to obtain mechanistic insight and information on molecular structure on the femtosecond timescale.
\end{abstract}




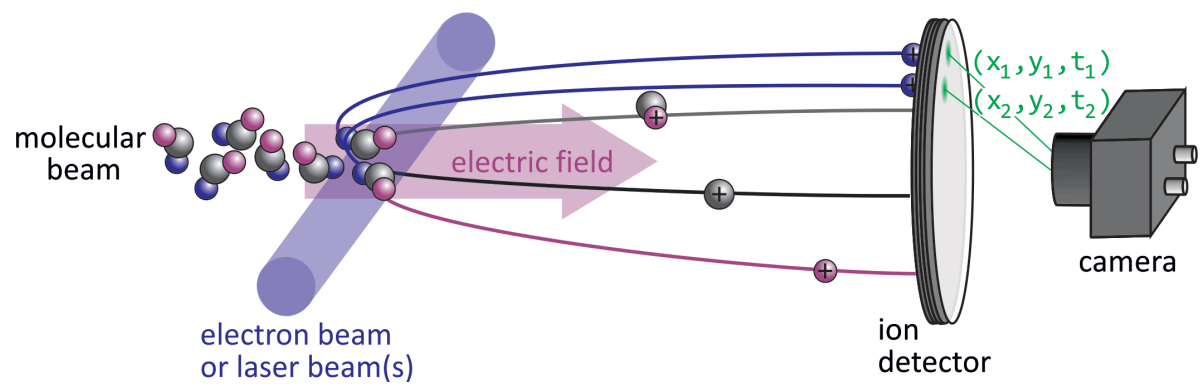

Figure 1: Schematic of a velocity-map imaging experiment for probing photoinduced processes. The reactant molecule of interest is prepared under vacuum in a molecular beam, which intersects with a pair of pulsed laser beams in the interaction region. The first laser pulse initiates the process of interest, while the second ionises the reaction products so that they can be detected. An electric field extracts the resulting ions along a flight tube to a position sensitive detector, where the scattering distribution is recorded. The detector comprises a pair of microchannel plates coupled to a phosphor screen, and converts each incoming ion to an optical signal that can be captured by a camera. Data are typically summed over many thousands of laser pump-probe cycles in order to capture enough events to generate relatively noise-free images of the scattering distribution.

\section{Introduction}

Chemical reaction dynamics studies probe chemical reactions on the scale of single reactive events, and can provide deep insight into both collision-induced and photoinitiated chemical processes. One of the key measurable quantities is the scattering distribution of the reaction products. The scattering distribution provides a unique 'fingerprint' for a given chemical process, and can be analysed to gain detailed information about the reaction mechanism, the reaction energetics, and the forces acting on the system at each stage of the process.

Up until the early 2000s, reaction dynamics studies were almost entirely confined to very small - three or four atom - chemical systems. However, recent developments in experimental techniques, theoretical methods, and computing power have made it possible to interrogate much larger chemical systems with relevance to a broad cross-section of chemists. In recent years, chemical dynamics studies have provided fundamental understanding of reaction processes relevant to atmospheric chemistry, combustion chemistry, interstellar chemistry, and biology. 


\subsection{Velocity-map imaging}

One of the key techniques that has driven progress in chemical reaction dynamics is velocity-map imaging ${ }^{1}$ (VMI). First developed in 1997 as a higherresolution version of its predecessor, ion imaging, ${ }^{2}$ velocity-map imaging allows measurement of the complete scattering distribution of a reaction product in a single measurement. The basic elements of a velocity-map imaging experiment for probing photon-induced processes, essentially a modified time-of-flight mass spectrometer, are shown in Figure 1. Velocity-map imaging instruments have been used to probe a wide variety of photoinitiated and collision-induced chemical processes, including photodissociation, photoionization, inelastic scattering, bimolecular reactions, Penning ionization, dissociative electron attachment, and electron ionization. ${ }^{3-6}$ With the achievable velocity resolution offering a quantum-state-resolved level of detail, such measurements have provided deep insights into some of the most fundamental processes in chemistry, in some cases even revealing previously undiscovered reaction mechanisms. ${ }^{7}$

A velocity-map imaging measurement provides two important pieces of information; the identity of the ions; and their scattering distributions:

1. All ions of a given charge $z$ receive the same amount of energy $z V$ from the electric field, where $V$ is the acceleration potential. Since the kinetic energy $K$ of the ions is given by $K=\frac{1}{2} m v^{2}$, the terminal velocity of the ions is inversely proportional to $\mathrm{m}^{1 / 2}$, and the ions separate in mass as they traverse the flight tube. The arrival time of a given ion at the detector then reveals its mass-to-charge ratio, $m / z$, and hence its identity.

2. The electric field is usually tuned such that the scattering distribution for each ion is 'crushed' into a two-dimensional projection at the detector plane. Various mathematical inversion algorithms ${ }^{8}$ are available to recover the full 3D distribution, with the caveat that most rely on the scattering distribution having an axis of cylindrically symmetry (this condition is satisfied in the majority of cases). Alternatively, the electric field can be tuned to stretch the ion cloud along the time-of-flight axis, in which case a suitably fast detector can record 'slices' through the scattering distribution. ${ }^{9-12}$ In either case, the $3 \mathrm{D}$ distribution can be recovered. The radial component of the distribution provides information on partitioning of the available energy between the various product degrees of freedom (translation, rotation, vibration, and electronic excitation). For example, one 
can infer that if the product translational energy (as measured in a VMI experiment) is low relative to the total available energy, then the internal energy is high, and vice versa. By considering correlations between the pump and probe laser polarisations and the transition dipole associated with the photoinitiation step, the angular distribution can provide information on the identity of the electronic states involved in the process, the timescale over which bond breaking occurs, and the nature of the forces acting in the transition state region of a reaction.

\subsection{Multi-mass velocity-map imaging}

In common with reaction dynamics studies more generally, for many years the focus for velocity-map imaging studies was on understanding very small chemical systems, containing perhaps up to five atoms, at extremely high levels of detail. This was driven partly by the need for high-level $a b$ initio potential energy surfaces to aid in interpretation of the data, and partly due to a widely-held belief that the rapid increase in the number of accessible states with molecular size would blur out any useful structure in the measured scattering distributions. These concerns proved to be unfounded, and there have since been many studies in which the photoproducts of significantly larger molecules have yielded highly structured - and therefore information-rich - images(see ${ }^{13-19}$ for a few examples). In parallel with experimental developments such as multimass velocitymap imaging, ${ }^{20}$ dramatic increases in available computational power have made it possible to calculate at least reduced-dimensionality potential energy surfaces for much larger molecules than had been accessible previously. Taken together, these advances have made it possible to perform detailed gas-phase chemical physics studies on chemical systems of real-world interest to a broad audience of chemists.

After a small amount of data processing, the data set from a multimass imaging experiment generally takes the form of a set of $(x, y, t)$ data points, encompassing the position $(x, y)$ and time-of-flight $t$ for each detected ion, on each experimental cycle. With suitable calibration of the velocity-map imaging setup, this can be transformed from the pixel-based $(x, y)$ units to $\left(v_{x}, v_{y}\right)$ velocity components for each ion. The data set can be summed over the $x$ and $y$ coordinates to obtain the total signal as a function of $t$, i.e. the time-of-flight

spectrum. From this, the mass-to-charge ratios and hence the identities of the products can be determined. Images of the scattering distribution for any de- 
sired mass-to-charge ratio can then be obtained by summing the $\left(v_{x}, v_{y}, t\right)$ data set over the relevant range of arrival times. In this way one can generate images of the scattering distribution for every fragment of interest. At this point, one often finds that it is not straightforward to unravel multiple different competing fragmentation pathways by looking at individual images. Attempts at identifying 'momentum matched' peaks within the velocity distributions of pairs of fragments can sometimes provide a 'way in' to this problem, but a more general and systematic approach is needed in all but the simplest of cases.

Ideally, we require a means of uncovering correlations between the scattering distributions of the various different products formed in the process under study. Covariance mapping offers a way to do just this. As we shall see, it allows us to determine the velocity distribution of one reaction product relative to the recoil direction of a second, 'reference' product. We shall show in the rest of this article that covariance mapping provides a powerful tool for unravelling complex reaction dynamics, and that in the special case in which the pump or probe laser initiates a Coulomb explosion, it also offers a direct route to obtaining information on molecular structure in the gas phase.

\section{Coincidence detection}

The idea of measuring correlations between reaction product velocities is not new, and before discussing covariance mapping in detail we should offer a brief overview of a technique that preceded covariance mapping and provides similar information, namely coincidence detection. As we shall see in Section 3, covariance mapping infers correlation between different experimental variables based on a statistical analysis of the data. In contrast, coincidence detection allows these correlations to be recorded directly for each event. Consider the example of a simple single photoionisation experiment, which produces an electron and an ion as detectable products (there may also be neutral products, depending on whether the nascent parent ion undergoes fragmentation). Typically, the electrostatic optics employed in such an experiment are configured such that positive and negative particles produced in the event of interest are accelerated in opposite directions towards a pair of detectors, one for positive particles and one for negative particles.

A key requirement for a coincidence approach is that fewer than one event of interest is recorded on each experimental cycle. If, on a particular cycle, 
an electron is detected at the 'negative particle' detector, the system is triggered to await the arrival of an ion at the 'positive particle' detector. If an ion is detected then both the electron and ion signals are stored; if not, the electron signal is discarded and the experiment resets for the next experimental cycle. Since the experiment is run with signal levels that ensure fewer than one event per cycle, we know that the detected electron and ion come from the same chemical event, and we therefore have a direct measurement of the way in which any measureable properties of the electron and ion are correlated for this event. The experiment is repeated over many experimental cycles in order to build up a statistical distribution of the correlation between the experimental variables of interest. Such an experiment is known as a 'photoelectron-photoion coincidence', or PEPICO experiment. ${ }^{21}$ Similar approaches can be applied to more complex photoionisation phenomena, yielding a variety of acronyms: PIPICO (photoion-photoion coincidence); PEPIPICO (photoelectron-photoion-photoion coincidence); PEPECO (photoelectron-photoelectron coincidence); and so on. ${ }^{22}$

By running such experiments at a high repetition rate, typically several kilohertz or higher, it is possible to achieve reasonable count rates and to acquire data within an experimentally tractable period of time, even with the extremely low signal level on each cycle. It is worth noting that if one attempts to run a coincidence experiment in a regime of higher counts per cycle then the approach rapidly breaks down, since the 'true coincidences' described above become lost amongst a background of 'false coincidences' between electrons and ions arising from different events. To illustrate this, consider an example in which we photoionise five molecules in a given cycle in the process $\mathrm{M}+h \nu \rightarrow \mathrm{M}^{+}+\mathrm{e}^{-}$. In this case we form five product pairs, which we can label $\left(\mathrm{e}_{1}^{-}, \mathrm{M}_{1}^{+}\right),\left(\mathrm{e}_{2}^{-}, \mathrm{M}_{2}^{+}\right)$, $\left(\mathrm{e}_{3}^{-}, \mathrm{M}_{3}^{+}\right),\left(\mathrm{e}_{4}^{-}, \mathrm{M}_{4}^{+}\right),\left(\mathrm{e}_{5}^{-}, \mathrm{M}_{5}^{+}\right)$. Electron $\mathrm{e}_{1}^{-}$has a true coincidence with ion $\mathrm{M}_{1}^{+}$, and measuring this coincidence signal provides useful information about the process under study. However, we would also measure false coincidences between the electron and the other four ions. These false coincidences contribute background 'noise' that tells us nothing about the process under study since the two particles were formed from different parent molecules.

Coincidence detection is widely used, and provides a powerful tool for unpicking the inner workings of molecular ionisation and dissociation processes. We refer the reader to a number of excellent reviews and books for further details and example applications, ${ }^{23-27}$ with apologies for any we have missed. Despite its successes, however, the requirement for very low counts per cycle mean that coincidence detection is not compatible with many state-of-the-art experiments, 
particularly those involving high-power or ultrafast lasers or those for which high repetition rates are not possible. It was to overcome these limitations that the covariance mapping approach was first developed.

\section{Covariance mapping}

Covariance mapping can provide similar information to that obtained in a coincidence experiment, but under conditions in which products are detected from multiple events on each experimental cycle. The time saving relative to coincidence detection depends on a number of factors, but for favourable systems (reasonably abundant product channels of interest and low noise levels), equivalent event statistics can be achieved using orders of magnitude fewer experimental cycles. ${ }^{28}$ We will focus on the application of covariance mapping to data sets from multi-mass velocity-map imaging experiments, in which case we are typically looking for correlations between the arrival times or the positions/velocities of the various detected ions.

Covariance is a statistical method that quantifies the correlation between two variables $A$ and $B$ in terms of the product of the deviation of each quantity from its mean value when averaged over a large number of measurements. If large values of $A$ tend to occur with large values of $B$, and/or small values of $A$ tend to occur with small values of $B$, then there will be a positive covariance between the two quantities. Conversely, if large values of $A$ tend to occur with small values of $B$, or vice versa, then the covariance will be negative. Formally, the covariance is defined as follows: ${ }^{29,30}$

$$
\begin{aligned}
\operatorname{Cov}(A, B) & =\langle(A-\langle A\rangle)(B-\langle B\rangle)\rangle \\
& =\langle A B\rangle-\langle A\rangle\langle B\rangle
\end{aligned}
$$

As noted above, the covariances that are most useful in the context of a multi-mass velocity-map imaging experiment are covariances between the arrival times of the various detected ions, and covariances between the recoil velocities of the ions. We will explore these in more detail in the following. 


\subsection{Time-of-flight covariance}

Covariances between the arrival times of different ions allow us to identify pairs of products formed from the same parent molecule. Examples include Frasinski and coworkers' ${ }^{29}$ pioneering investigation into the multiphoton ionisation and fragmentation of $\mathrm{CO}_{2}$, and Bhardwaj et al's study into the dissociative ionization of benzene. ${ }^{31}$ Additional examples can be found in the excellent recent review by Frasinski, ${ }^{32}$ which is recommended to the interested reader.

To determine the time-of-flight ( ToF) covariance, we calculate the covariance between the reaction product time-of-flight spectrum and itself, i.e. the covariance is calculated between each pair of time points in the ToF spectrum recorded on a given experimental cycle, with the averaging being performed over the number of experimental cycles. This yields a two-dimensional covariance matrix which can be plotted in the form of a covariance map. An example is shown for the fragmentation products of doubly-charged $\mathrm{CF}_{3} \mathrm{I}^{2+}$ ions ${ }^{33}$ in Figure 2. Since every ion is automatically covariant with itself, there is a line of 'auto-covariance' along the diagonal, which is simply the variance of the time-offlight spectrum. The covariance signals of interest, which contain information about the fragmentation mechanism, are the 'cross peaks' that appear away from the diagonal.

The information contained in a ToF-ToF covariance map is two-fold. Firstly, the presence of a cross peak connecting two different product ions indicates that they were formed together in the same event, i.e. that the two fragments originate from the same parent molecule. Secondly, the two-dimensional shape of the cross peak reveals information on the relative momenta of the two ions, yielding insight into the fragmentation mechanism. We can illustrate this using the ToF-ToF covariance map for $\mathrm{CF}_{3} \mathrm{I}$ shown in Figure 2.

Our starting point is to recognise that the velocity component $v_{z}$ along the time-of-flight axis for any given fragment is the sum of the velocity component arising from the fragmentation process of interest and the velocity acquired as the ion is accelerated towards the detector by the electric field from the time-offlight ion optics. The latter determines the position of the centre of the fragment time-of-flight peak, while the distribution of the former determines the width and peak shape. Assuming a uniform acceleration potential $V$, for an ion of mass $m$ and charge $z$ traversing a flight tube of length $d$, we have

$$
v_{z}=\left(\frac{2 z V}{m}\right)^{1 / 2}+u \cos \theta
$$




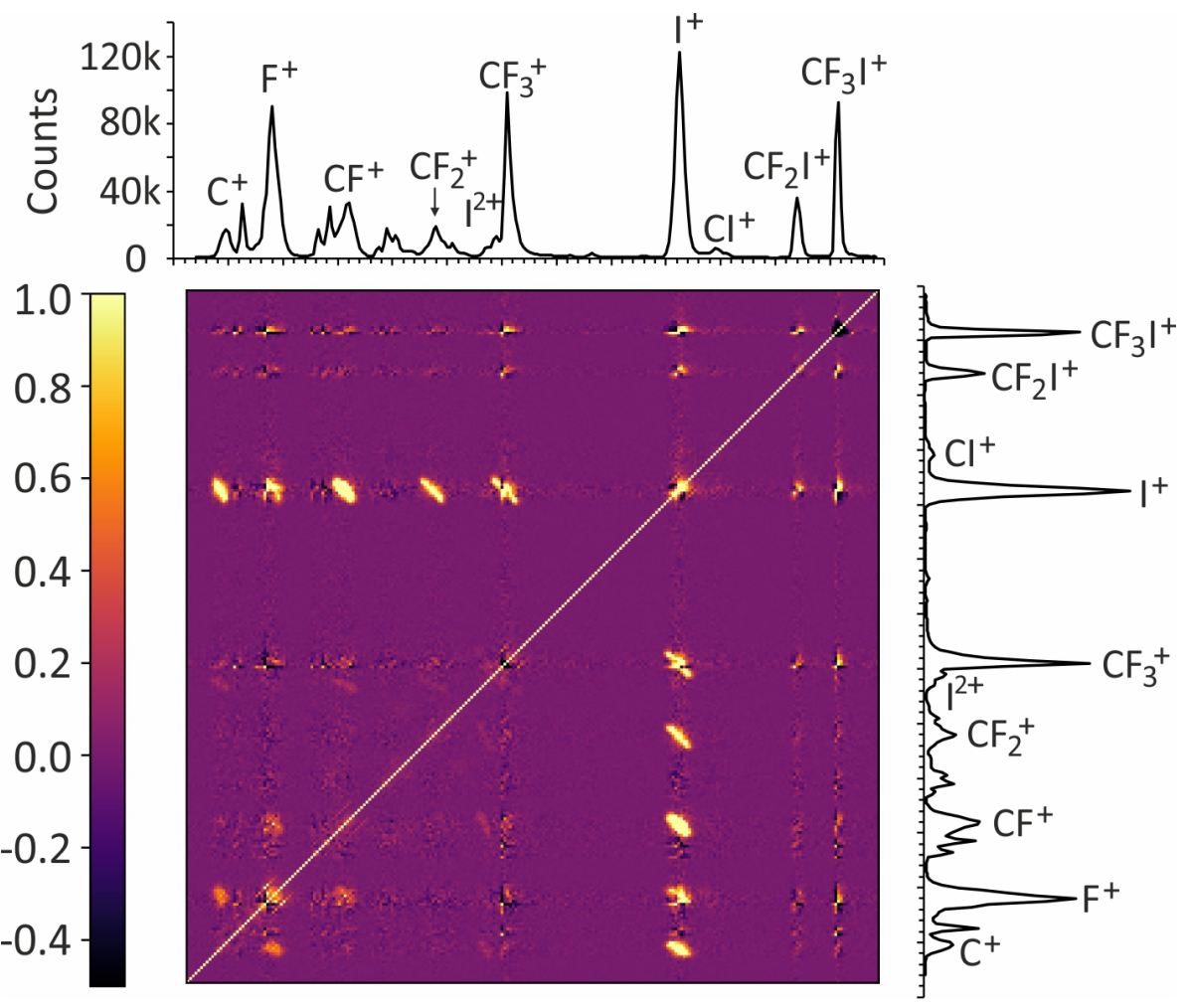

Figure 2: Time-of-flight covariance map for the ionic products formed in the $100 \mathrm{eV}$ electron ionization of $\mathrm{CF}_{3} \mathrm{I}$. Adapted from reference. ${ }^{33}$

where $\mathbf{u}$ is the velocity imparted to the fragment as a result of the dissociation process, $u$ is the magnitude of this velocity, and $\theta$ is the angle between $\mathbf{u}$ and the time-of-flight axis. The flight time of the ion is therefore

$$
t=\frac{d}{v_{z}}=d\left[\left(\frac{2 z V}{m}\right)^{1 / 2}+u \cos \theta\right]^{-1}
$$

The centre of the time of flight peak, $t_{0}$, is determined by the velocity contribution described in the first term in Equation 3.

$$
t_{0}=d\left(\frac{m}{2 z V}\right)^{1 / 2}
$$

By taking the difference between Equations 4 and 5, after some algebra and with the (small) approximation that the first term in Equation 3 is much larger 
than the second term, we can determine a simple expression for the deviation from the centre of the time-of-flight peak for a given ion.

$$
\begin{aligned}
\Delta t & =t-t_{0} \\
& =-\frac{d m u}{2 z V} \cos \theta \\
& =-k p \cos \theta
\end{aligned}
$$

where $p=m u$ is the ion momentum arising from the dissociation process, and $k=d /(2 z V)$ is a constant depending only on instrument parameters and the charge of the ion.

We have therefore shown that the deviation $\Delta t$ of an ion's time of flight from $t_{0}$ is proportional to the momentum component $p \cos \theta$ of the ion along the time-of-flight axis. In a ToF covariance map, each cross peak is essentially a plot of $\Delta t_{1}$ vs $\Delta t_{2}$ for a pair of ions. The gradient of the cross-peak signal therefore provides information on the relative momenta of the two ions, which can be used to determine the fragmentation mechanism.

To illustrate these ideas, we will consider the dissociation of a doubly-charged ion $\mathrm{ABC}^{2+}$ via a number of different mechanisms. We first consider a simple two-body dissociation, $\mathrm{ABC}^{2+} \rightarrow \mathrm{A}^{+}+\mathrm{BC}^{+}$. In this case the two product ions $\mathrm{A}^{+}$and $\mathrm{BC}^{+}$must be formed with equal and opposite momenta, and the slope of the cross-peak is therefore -1 . This is the case for the cross-peak arising from the $\mathrm{CF}_{3}^{+}+\mathrm{I}^{+}$products from $\mathrm{CF}_{3} \mathrm{I}^{2+}$ dissociation in Figure 2 .

Three body dissociations may occur via at least three different mechanisms, often referred to in terms of 'concerted', 'initial charge separation', and 'deferred charge separation' processes. The first of these is a single-step process, while the latter two involve two steps.

$$
\begin{aligned}
\text { Concerted : } & \mathrm{ABC}^{2+} \longrightarrow \mathrm{A}^{+}+\mathrm{B}^{+}+\mathrm{C} \\
\text { Initial charge separation : } & \mathrm{ABC}^{2+} \longrightarrow \mathrm{A}^{+}+\mathrm{BC}^{+} \longrightarrow \mathrm{A}^{+}+\mathrm{B}^{+}+\mathrm{C} \\
\text { Deferred charge separation : } & \mathrm{ABC}^{2+} \longrightarrow \mathrm{AB}^{2+}+\mathrm{C} \longrightarrow \mathrm{A}^{+}+\mathrm{B}^{+}+\mathrm{C}
\end{aligned}
$$

In the case of the concerted mechanism, the neutral product $\mathrm{C}$ can often be treated as a spectator and assumed to be formed with very little momentum relative to the charged fragments $\mathrm{A}^{+}$and $\mathrm{B}^{+}$. In this case $\mathbf{p}_{\mathrm{A}^{+}} \approx-\mathbf{p}_{\mathrm{B}^{+}}$and the cross peak between $\mathrm{A}^{+}$and $\mathrm{B}^{+}$will have a slope very close to -1 , similar to the cross peak between $\mathrm{A}^{+}$and $\mathrm{BC}^{+}$in the two-body dissociation. 
The situation becomes somewhat more complex in the case of two-step dissociations, but by considering the momenta imparted to the relevant fragments in each of the two steps it is possible to obtain expressions for the slopes of the relevant cross peaks in the ToF covariance plot, as well as to make predictions about the peak shapes. The expressions depend simply on the masses of the various species involved in each mechanism, so by fitting the slopes of the cross peaks it is often possible to determine which of the mechanisms is dominant. These cases are treated in detail by Eland and coworkers ${ }^{34,35}$ in the context of PEPIPICO measurements on a number of small molecules.

\subsection{Recoil-frame covariance-map images}

We have shown how ToF-ToF covariance signals can be used to identify pairs of fragments formed via a particular reaction mechanism, as well as to obtain information on the relative momentum projections of the fragments onto the time-of-flight axis, $z$. Velocity-map imaging provides information on the other two momentum components, in the $x y$ plane, and covariances between these components provide further information on the dynamics of the process of interest. ${ }^{36-40}$

The complete covariance matrix between a pair of two-dimensional velocitymap images would have four dimensions. However, we can generate more intuitively useful two-dimensional covariance maps from the images as follows. When considering the covariance between two different ions, we designate one to be the 'reference' species and the second to be the 'signal' species. The goal is to find any correlations that exist between the signal intensities within each pixel of the velocity-map images for the two ions. The covariance is therefore calculated between each pixel in the reference ion image and each pixel in the signal ion image, with the averaging performed over the acquisition cycles. This process yields a separate covariance map for each pixel in the reference image, showing correlations between this pixel and all of the pixels in the signal image. To generate the overall covariance map, each individual map is rotated to align all of the reference pixels along the same direction, and the individual covariance maps for each reference pixel are then summed ${ }^{1}$. Any positive regions within the resulting covariance map can be interpreted approximately as showing the

\footnotetext{
${ }^{1}$ For reasons of computational efficiency, in practice the rotation step is performed before the covariance calculation in order to allow the individual covariance matrices to be summed as they are calculated. This avoids having to store the full $4 \mathrm{D}$ covariance map at any point in the calculation.
} 
(projected) velocity distribution of the signal ion relative to the recoil direction of the reference ion, i.e. the relative velocity distribution of the two ions. We note that the total intensity of the recoil-frame covariance map image for a given ion pair is equal to the total intensity of the corresponding cross-peak in the ToF covariance map discussed in Section 3.1.

As an 'extra for experts', we note that because both of the images used to generate the covariance map are $2 \mathrm{D}$ projections of the respective $3 \mathrm{D}$ fragment velocity distributions, we have no information on the $z$ (ToF axis) component of the reference ion velocity. As a result, the covariance map is therefore the $2 \mathrm{D}$ projection of covariance signals for all reference directions in three dimensions that project onto the chosen reference direction in the $2 \mathrm{D}$ plane. These span a 180 degree arc bisected by the image plane and result in a characteristic 'tail' on the covariance signals extending towards the centre of the covariance-map images. We shall see in Section 3.3.3 that this signal averaging can be eliminated by performing $3 \mathrm{D}$-sliced velocity-map imaging measurements, in which case the rotation can be performed in three dimensions to align all of the reference ion velocities along a single axis. However, for the moment we will focus on the 2D covariance maps obtained as described above from a pair of crushed velocitymap images.

\subsubsection{Two-body dissociations}

We can illustrate some of the features of recoil-frame covariance maps through a series of simple simulations. We first consider the case of a two-body dissociation $\mathrm{AB}^{2+} \rightarrow \mathrm{A}^{+}+\mathrm{B}^{+}$. We have run simulations to model a number of different experimental scenarios in which fragmentation products from between one and ten parent molecules per experimental cycle were detected. Detection efficiencies were varied between $50 \%$ and $100 \%$ and data were simulated for 100,000 cycles. In each event, a momentum-matched $\mathrm{A}^{+}+\mathrm{B}^{+}$ion pair was generated, with relative velocities of 100 and 80 pixel units (px), respectively. The direction of the relative velocity vector was chosen randomly for each trajectory. The resulting covariance images contain regions of positive and negative covariance (the interpretation of negative covariance will be discussed shortly), and were normalised to unit positive covariance for ease of comparison. As expected, the results were found to be essentially identical for each set of conditions used, with the detection efficiency and number of dissociating molecules affecting only the total image intensity before normalisation. A typical set of simulation results 

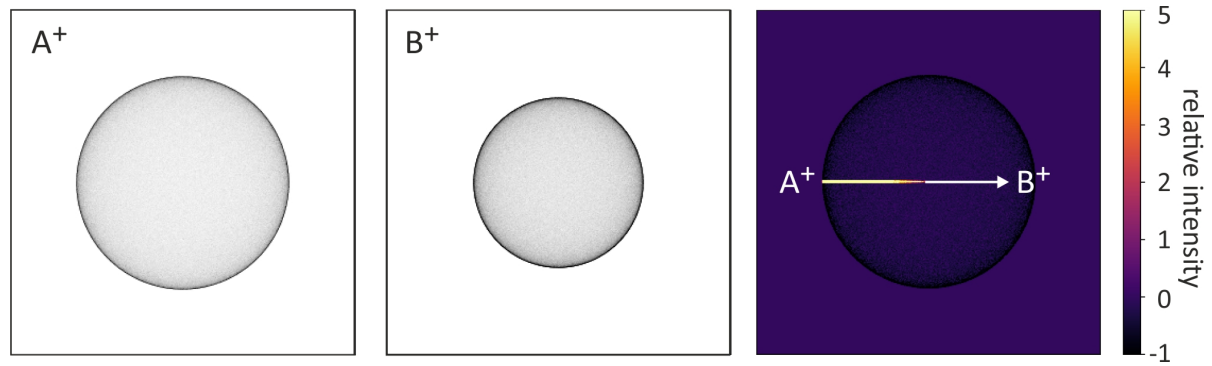

Figure 3: Simulated velocity-map images for the $\mathrm{A}^{+}$and $\mathrm{B}^{+}$products of $\mathrm{AB}^{2+}$ dissociation, together with the recoil-frame covariance-map image for the $\mathrm{A}^{+}$ product relative to the reference direction defined by the $\mathrm{B}^{+}$velocity vector.

is displayed in Figure 3, showing the simulated velocity-map images for $\mathrm{A}^{+}$ and $\mathrm{B}^{+}$, together with the covariance map for $\mathrm{A}^{+}$when $\mathrm{B}^{+}$is defined as the reference ion.

Since the $\mathrm{A}^{+}$and $\mathrm{B}^{+}$ions are formed with well defined speeds but randomly selected angles, the individual velocity-map images both take the form of a sphere projected onto a plane, with radius 100 pixels for $\mathrm{A}^{+}$and 80 pixels for $\mathrm{B}^{+}$. The covariance map reveals the correlation between the velocity vectors of the two ions, displaying strong positive covariance in the direction opposite to that of the recoil ion, with negative covariance in all other regions. Since we are viewing a $2 \mathrm{D}$ projection of the covariance between the two velocity vectors, the positive signal appears as a line rather than a single point. If instead of simulating crushed velocity-map images we had limited the simulation to two dimensions (equivalent to simulating a sliced imaging experiment), the covariance signal would be reduced to a single point at the radius corresponding to the $\mathrm{A}^{+}$velocity, with a well-defined ring of negative covariance in all other regions at the same radius. The same would also be true if we had simulated an experiment in which the dissociating molecules were pre-aligned along a laboratory-frame axis.

Various interpretations of negative covariance have been reported in the literature, ${ }^{32,41-45}$ and considerable confusion has arisen over its meaning. In the present context it can be understood as follows. The covariance map reveals whether the probability of finding a signal in a given pixel in the 'signal' image increases or decreases relative to the mean when a signal is present in a given pixel of the 'reference' image. The mean intensity in each pixel of the signal image is simply the signal observed in the velocity-map image from which the 
covariance map is constructed. Once the data from each experimental cycle have been rotated such that the reference ion velocity lies along the chosen reference direction, we are more likely to find the partner ion velocity in the opposite direction from the reference than anywhere else, while in all other directions there are fewer ions found than in the mean image. This results in the regions of positive and negative covariance observed. It should be noted that these arguments only apply within a covariant channel. For example, if we had the possibility of two competing channels, such as a dication $\mathrm{ABC}^{2+}$ dissociating to give either $\mathrm{A}^{+}+\mathrm{B}^{+}+\mathrm{C}$ or $\mathrm{A}^{+}+\mathrm{B}+\mathrm{C}^{+}$then we can consider covariances between the ion pairs $\left(\mathrm{A}^{+}, \mathrm{B}^{+}\right),\left(\mathrm{A}^{+}, \mathrm{C}^{+}\right)$, and $\left(\mathrm{B}^{+}, \mathrm{C}^{+}\right)$. The above arguments would apply to the first two of these pairs, since each pair of ions are formed together in the same process. The $\left(\mathrm{A}^{+}, \mathrm{B}^{+}\right)$covariance would reveal information on the first of the two pathways, but nothing about the second pathway, and vice versa for the $\left(\mathrm{A}^{+}, \mathrm{C}^{+}\right)$covariance. However, we would not expect to see any covariance signal, positive or negative, between $\mathrm{B}^{+}$and $\mathrm{C}^{+}$since they are not formed in the same process (i.e. cannot be formed together from a given parent ion) and therefore their velocities cannot be correlated with each other.

\subsubsection{Three-body dissociations}

We now move on to consider the recoil-frame covariance maps that we might expect for the various three-body dissociation pathways outlined previously in Section 3.1, i.e. the concerted, initial charge separation, and deferred charge separation mechanisms. As noted earlier, in the concerted pathway $\mathrm{ABC}^{2+} \rightarrow$ $\mathrm{A}^{+}+\mathrm{B}^{+}+\mathrm{C}$, the majority of the kinetic energy is released into the two charged fragments, and it is often a good approximation to treat the neutral fragment as a 'spectator'. In this case the covariance images between $\mathrm{A}^{+}$and $\mathrm{B}^{+}$will be very similar to those described above for the two-body dissociation case. If significant momentum is transferred to the neutral fragment $\mathrm{C}$ during the dissociation then this will have the effect of blurring the correlation somewhat. However, we would still expect the two charged fragments to recoil at almost 180 degrees to one another.

Figure 4 shows velocity-map images and covariance-map images resulting from simulations of the two possible two-step mechanisms. In both cases the ions are allowed to dissociate in any orientation in each of the two steps. In the simulation of the initial charge separation process, $\mathrm{ABC}^{2+} \rightarrow \mathrm{A}^{+}+\mathrm{BC}^{+} \rightarrow$ $\mathrm{A}^{+}+\mathrm{B}^{+}+\mathrm{C}$, the $\mathrm{A}^{+}$and $\mathrm{BC}^{+}$products of the first step are given velocities 
(a) Initial charge separation
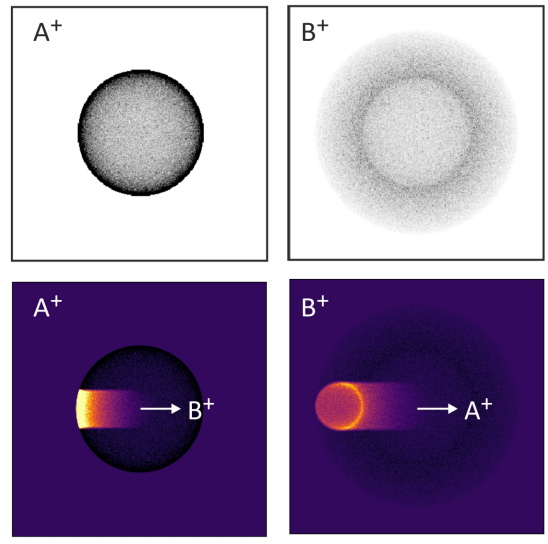

(b) Deferred charge separation
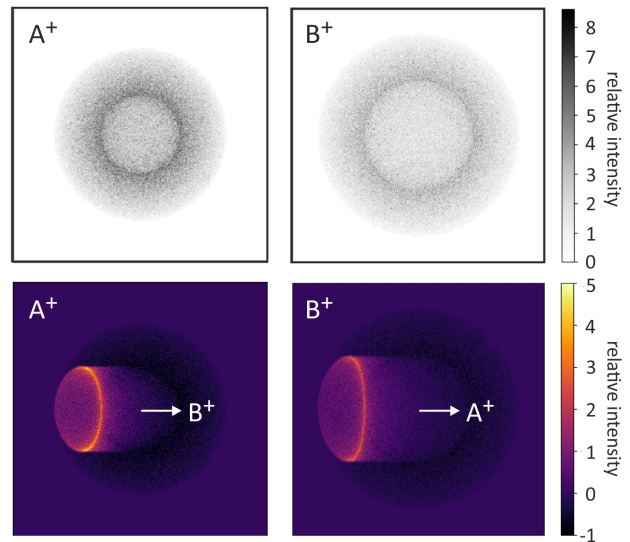

Figure 4: Simulated velocity-map images (top row) and covariance-map images (bottom row) for the ionic products of the two possible two-step dissociation mechanisms for $\mathrm{ABC}^{2+}$ : (a) initial charge separation, $\mathrm{ABC}^{2+} \rightarrow \mathrm{A}^{+}+\mathrm{BC}^{+} \rightarrow$ $\mathrm{A}^{+}+\mathrm{B}^{+}+\mathrm{C}$; (b) deferred charge separation $\mathrm{ABC}^{2+} \rightarrow \mathrm{AB}^{2+}+\mathrm{C} \rightarrow \mathrm{A}^{+}+\mathrm{B}^{+}+\mathrm{C}$.

(in pixel units) of 80 and $100 \mathrm{px}$, respectively, and the $\mathrm{B}^{+}$final product is given an additional randomly-directed velocity in the second step of $30 \mathrm{px}$. For the deferred charge separation process, $\mathrm{ABC}^{2+} \rightarrow \mathrm{AB}^{2+}+\mathrm{C} \rightarrow \mathrm{A}^{+}+\mathrm{B}^{+}+\mathrm{C}$, the $\mathrm{AB}^{2+}$ ion formed in the first step is given a velocity of $30 \mathrm{px}$, and the $\mathrm{A}^{+}$and $\mathrm{B}^{+}$products of the second step are given additional velocities of 80 and $100 \mathrm{px}$, respectively. Simulations were performed over 100,000 cycles, with five $\mathrm{ABC}^{2+}$ parent dications dissociating on each cycle.

For these simple simulations in which each step in the mechanism results in a single well-defined kinetic energy release, the velocity-map images for the $\mathrm{A}^{+}$and $\mathrm{B}^{+}$products provide a clear distinction between the two pathways even before performing the covariance analysis. The $\mathrm{A}^{+}$product of the initial charge separation pathway is formed in a single step with a single velocity, and therefore appears as a sharp ring (projection of a spherical shell) in the velocity-map image. The $\mathrm{B}^{+}$product is the result of two dissociation steps, and relative to the $\mathrm{A}^{+}$image, every point on the ring is 'blurred' by the momentum 'kick' in a random direction associated with loss of the neutral $\mathrm{C}$ product in the second step of the mechanism. In the deferred charge separation mechanism, the neutral is lost first, and so in this case both the $\mathrm{A}^{+}$and $\mathrm{B}^{+}$velocity distributions are 'blurred' by the momentum gained during this neutral loss step. It is worth noting at this point that in most real systems, each step in the mechanism 
would yield a distribution of kinetic energy releases, blurring the well-defined ring observed for the $\mathrm{A}^{+}$product in the initial charge separation case, and making the distinction between the two mechanisms much less clear on the basis of the velocity-map images alone.

We now consider the covariance-map images for the two cases. We see that the two mechanisms give very distinctive covariance maps, which are immediately distinguishable from each other. As in the two-body dissociation case, there are regions of positive and negative covariance, as well as a 'tail' towards the centre of the images resulting from the two-dimensional projection. Consider first the initial charge separation pathway. The form of the covariance maps can be understood by comparison with those observed previously for a two-body dissociation. The covariance map for $\mathrm{B}^{+}$relative to $\mathrm{A}^{+}$reveals a (projected) sphere of radius $30 \mathrm{px}$ centred at the $100 \mathrm{px}$ velocity of the $\mathrm{BC}^{+}$ intermediate, providing a fairly direct visualisation of the energetics for both dissociation steps. The covariance image of $\mathrm{A}^{+}$relative to $\mathrm{B}^{+}$is slightly less intuitive to understand, appearing as an arc on a circle of radius $80 \mathrm{px}$, the velocity imparted to the $\mathrm{A}^{+}$product in the first step in the mechanism. The fact that an arc is observed rather than a point is due to the subsequent dissociation of the $\mathrm{BC}^{+}$partner fragment, the result of which is that the two ions are no longer confined to lie only along the initial dissociation vector.

In the case of the deferred charge separation mechanism, both covariance maps reveal an oval-shaped distribution. The first dissociation step yields $\mathrm{AB}^{2+}$ ions on the surface of a Newton sphere, which subsequently undergo further dissociation along a random relative velocity vector into the observed $\mathrm{A}^{+}+\mathrm{B}^{+}$ products. The oval appearance of the covariance map arises from the rotation of the covariance maps for individual pixels (see Section 3.2) into the reference frame, in which the reference ion lies along the $x$ axis.

These simulations show that recoil-frame covariance-map imaging provides a new way to distinguish between various possible reaction mechanisms. In real applications, the fragment velocities associated with each dissociation step are often not known, and span a distribution rather than taking a single value. However, a combination of simulation and experiment can provide considerable insight into the reaction dynamics in complex systems. 


\subsection{More sophisticated covariance mapping techniques}

For the majority of this review, we will focus on applications of the relatively straightforward two-body covariance-map imaging methods described above. However, for completeness we should mention some of the more sophisticated covariance mapping techniques that are starting to be used in a variety of applications. These include partial covariance, higher-order covariances, and threedimensional recoil frame covariance mapping.

\subsubsection{Partial covariance and contingent covariance mapping}

So far we have assumed that the only circumstance in which we will see a covariance signal between two ions is when they are formed together in a particular chemical process. This would be the case in a 'perfect' experiment in which all experimental parameters that might affect signal intensities (laser pulse energies, molecular beam intensities, etc) could be kept constant. However, in a real experiment these parameters often fluctuate between experimental cycles and/or drift over longer timescales. This can lead to false covariances in the data set that do not reflect any correlations intrinsic to the chemical system of interest. For example, if the molecular beam intensity or a laser pulse energy decreases over time in a velocity-map imaging experiment, then signals from all

of the detected ions would also decrease over time. A reduction in the intensity of one ion would correlate with a reduction in intensity of all of the other ions, and all of the ion signals would become covariant with each other, regardless of the chemical process in which they are formed.

If the fluctuating parameter $P$ can be measured on each experimental cycle, then covariance maps can be corrected for correlations of this kind using the method of partial covariance. By generating covariance matrices between the signals of interest and the fluctuating parameter and subtracting these from the covariance matrix of interest, the true covariance is revealed. The resulting expression is ${ }^{32,46,47}$

$$
\operatorname{pCov}(A, B ; P)=\operatorname{Cov}(A, B)-\frac{\operatorname{Cov}(A, P) \operatorname{Cov}(P, Y)}{\operatorname{Cov}(P, P)}
$$

Here, $\mathrm{p} \operatorname{Cov}(A, B ; P)$ is the partial covariance matrix, which should now be independent of the fluctuating experimental parameter $P$, and the other covariances are as defined previously in Equation 2. A similar analysis can also be carried out when there are multiple fluctuating parameters. ${ }^{48}$ 
Partial covariance works well when there is a linear dependence between the signals of interest and the fluctuating experimental parameter. However, this is often not the case, e.g. fluctuations in the intensity of a laser beam that initiates a two-photon process will lead to fluctuations in the signal that depend quadratically on the laser intensity. In such cases, an approach known as contingent covariance mapping, first introduced by Zhaunerchyk et al, ${ }^{48}$ can provide a better correction to the data set. A histogram of the fluctuating experimental parameter is used to group the data set into a number of subsets within which the parameter is approximately constant. Covariance maps are calculated for each subset, and then the individual covariance maps are summed, with each contribution appropriately weighted according to the fraction of the data set represented by each subset. In this way, false covariances due to the fluctuating parameter are avoided. The contingent covariance approach can also be extended to cover situations in which more than one parameter fluctuates.

\subsubsection{Higher order covariances}

So far we have only considered covariance between two variables, i.e. two-fold covariance. Zhaunerchyk and coworkers ${ }^{48}$ have shown that in certain cases it is also possible to investigate three-variable (three-fold) covariances, defined by analogy with the two-variable covariance in Equation 2 as

$$
\operatorname{Cov}(A, B, C)=\langle(A-\langle A\rangle)(B-\langle B\rangle)(C-\langle C\rangle)\rangle
$$

For this approach to be valid, it is necessary to show not only that non-zero covariances arise when the three variables are correlated, but also that the covariance is zero when two or three of the variables are independent. Zhaunerchyk and coworkers showed that both of these requirements are met for processes

which are governed by Poisson distributions, in which case the physical meaning of two-fold and three-fold covariance is the same. It is also possible to perform a partial covariance analysis for three variables. However, when attempting to extend the formalism to four-variable covariance it was shown that pairwise correlations amongst four variables can lead to a non-zero 'four-fold' covariance, so that a simple extension of Equation 10 to four variables is not valid.

We note that observing three-body covariances experimentally requires a high detection efficiency. For example, consider an experiment probing a threebody breakup, leading to products $\mathrm{A}+\mathrm{B}+\mathrm{C}$. If the detection efficiency for each fragment is $50 \%$, as is typical for commonly-used microchannel plate detectors, 
then whenever we detect an A fragment, we have a $50 \%$ chance of also detecting $\mathrm{B}$ or $\mathrm{C}$, and only a $25 \%$ chance of detecting A, B, and C. Only $25 \%$ of the signal can therefore reveal the three-body covariances of interest, with the situation quickly degenerating further as the detection sensitivity drops. Nevertheless, there are a number of demonstrations of three-fold covariance-map imaging. For example, three-fold time-of-flight covariance was used as long ago as 1991 by Frasinski et $a l^{49}$ to uncover correlations between triplets of ions formed in the dissociation of multiply-charged $\mathrm{N}_{2} \mathrm{O}$. It was also used by Bryan et al.,${ }^{50}$ alongside two-fold covariance mapping, to reveal a large number of two-body and three-body Coulomb explosion channels in the dissociation of multiply charged carbonyl sulphide, OCS.

Three-fold recoil-frame covariance mapping has also been demonstrated by a number of authors. Of particular note, three-fold covariance mapping applied to Coulomb explosion products of chiral molecules can be used to differentiate between enantiomers. This was highlighted by Christensen et al. ${ }^{51}$ and by Pickering et al. ${ }^{52}$ in their recent studies of a substituted biphenyl molecule. This first use of covariance mapping of Coulomb explosion products to establish the absolute configuration of a molecule followed relatively quickly on the heels of an earlier study by Pitzer et al. ${ }^{53}$ who demonstrated a similar result for a chiral methane derivative using $3 \mathrm{D}$ coincidence imaging.

\subsubsection{Three-dimensional recoil-frame covariance mapping}

In Section 3.2 we looked in some detail at the information contained in twodimensional recoil-frame covariance-map images, generated from pairs of crushed velocity-map images. It is also possible to generate three-dimensional covariance maps from a pair of three-dimensional velocity-map images recorded using slice imaging. 3D covariances of this type have been demonstrated recently by Lee $e t$ $a l^{20,54}$ for various Coulomb explosion products of multiply-charged $\mathrm{CF}_{3} \mathrm{I}$ ions. An example is shown in Figure 5, which shows 3D recoil-frame covariances for various subsets of the two-body dissociation products $\mathrm{CF}_{3}^{+}$and $\mathrm{I}^{+}$. As expected, the two ions recoil in opposite directions in three dimensions. We note that for this simple two-body dissociation, the central slices through the two 3D scattering distributions - as obtained in a more conventional sliced VMI experiment - contain all of the required information on the relative velocities of the two fragments. However, this would not be the case for a many-body dissociation in which any of the fragments has an out-of-plane velocity component. 

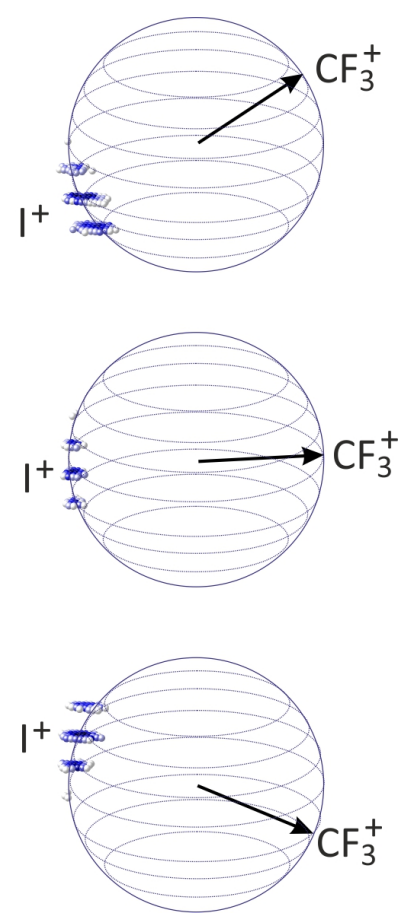

Figure 5: 3D covariance-map images for the $\mathrm{CF}_{3}^{+}+\mathrm{I}^{+}$Coulomb explosion products of $\mathrm{CF}_{3} \mathrm{I}^{2+}$. The direction of travel of the $\mathrm{CF}_{3} \mathrm{I}$ reference ion is shown by the black arrow, and the velocity distribution of the $\mathrm{I}^{+}$ion relative to this direction is shown by the intensity distribution within each slice of the 3D covariance map. Adapted from reference ${ }^{54}$.

Three-dimensional covariance mapping will almost certainly soon be combined with three-fold covariance mapping, as described in the previous Section, in order to investigate covariant relationships between three reaction products in three dimensions rather than two. This is likely to prove particularly useful in analysing chiral data sets, since it will in principle provide a very direct way in which to disentangle contributions from each handedness.

\section{Applications of recoil-frame covariance-map imag- ing}

Having introduced the principles underlying recoil-frame covariance map imaging, we are now ready to consider recent applications of the technique within a variety of chemical dynamics studies. For a summary of earlier studies exploit- 
ing time-of-flight covariance mapping, we again refer the reader to the recent review by Frasinski. ${ }^{32}$

\subsection{Dissociation dynamics of doubly and triply charged ions: disentangling sequential dissociation pathways}

\subsubsection{Electron ionisation of $\mathrm{CF}_{3} \mathrm{I}$}

In Section 3.1 we considered as an example the time-of-flight covariance map of the various fragment ions formed in the dissociative electron ionization of $\mathrm{CF}_{3} \mathrm{I}$ at an electron energy of $100 \mathrm{eV}$. At this energy, the vast majority of collision events yield a singly charged parent ion, which often dissociates into a singly charged daughter ion and a neutral. ${ }^{55}$ However, since only the ionic fragment is detected, covariance analysis is blind to these major channels, and allows the products of less common processes involving doubly and triply charged parent ions to be observed even in the presence of large signals from singly-charged pathways. ${ }^{33,40}$ The ToF covariance map shown in Figure 2 shows strong covariance signals involving the ion pairs $\left(\mathrm{I}^{+}, \mathrm{CF}_{3}^{+}\right),\left(\mathrm{I}^{+}, \mathrm{CF}_{2}^{+}\right),\left(\mathrm{I}^{+}, \mathrm{CF}^{+}\right),\left(\mathrm{I}^{+}, \mathrm{C}^{+}\right)$, $\left(\mathrm{F}^{+}, \mathrm{I}^{+}\right)$, all arising from the dication $\mathrm{CF}_{3} \mathrm{I}^{2+}$, and much weaker signals for the corresponding pairs involving $\mathrm{I}^{2+}$ rather than $\mathrm{I}^{+}$. The presence of these signals reveals a significant number of decay channels for the dication and trication. These include two-body decay to form a methyl and iodide ion pair, alongside a number of many-body decay channels involving loss of one or more neutral fluorine atoms. Recoil-frame covariance-map images provide further insight into each of these pathways. Figure 6 shows recoil-frame covariance maps for a subset of the ion pairs arising from fragmentation of the dication. Similar distributions are observed for fragmentation of the trication, though the products appear at larger radii due to the greater Coulomb repulsion during the early stages of the fragmentation.

The first covariance map shown in Figure 6 corresponds to the two-body dissociation channel, and clearly shows the pair of ions recoiling in opposite directions in accordance with conservation of momentum. The remaining images correspond to the channels: 

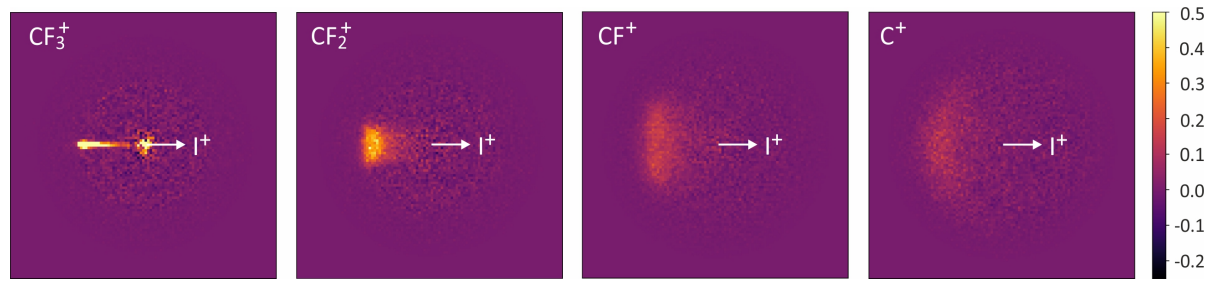

Figure 6: Covariance-map images for the dissociation products of $\mathrm{CF}_{3} \mathrm{I}^{2+}$. In each case the arrow marks the reference ion and the label on the top left of the image denotes the signal ion. Adapted with permission from reference. ${ }^{33}$

$$
\begin{array}{lll}
\mathrm{CF}_{3} \mathrm{I}^{2+} & \longrightarrow \mathrm{CF}_{2}^{+}+\mathrm{I}^{+}+\mathrm{F} \\
\mathrm{CF}_{3} \mathrm{I}^{2+} & \longrightarrow \mathrm{CF}^{+}+\mathrm{I}^{+}+2 \mathrm{~F} \\
\mathrm{CF}_{3} \mathrm{I}^{2+} & \longrightarrow \mathrm{C}^{+}+\mathrm{I}^{+}+3 \mathrm{~F}
\end{array}
$$

As noted in Section 3.2.2, these many-body channels may proceed by a number of different mechanisms involving fragmentation of the doubly-charged ion into two singly charged ions and loss of one or more fluorine atoms. In all of the covariance-map images, we see that the two charged fragments recoil predominantly in opposite directions. However, the momentum 'kick' imparted to the products of $\mathrm{C}-\mathrm{F}$ bond fission causes the correlation to become less well defined with each successive $\mathrm{F}$ atom loss.

The covariance-map images can provide insight into the detailed mechanism of the various dissociation processes. Take for example the first of the three channels shown in Equation 11. As explained in Section 3.2.2, there are three possible dissociation mechanisms:

1. Concerted dissociation of $\mathrm{CF}_{3} \mathrm{I}^{2+}$ into $\mathrm{CF}_{2}^{+}+\mathrm{I}^{+}+\mathrm{F}$;

2. Initial charge separation into $\mathrm{CF}_{3}^{+}+\mathrm{I}^{+}$, followed by loss of neutral $\mathrm{F}$ from $\mathrm{CF}_{3}^{+}$;

3. Loss of a neutral $\mathrm{F}$ atom from the parent $\mathrm{CF}_{3} \mathrm{I}^{2+}$ ion, followed by charge separation to form the final products.

Previous work by other authors, in which $\mathrm{CF}_{3} \mathrm{I}^{2+}$ ions were formed either by photoionization or electron ionization, has indicated that at low electron energies $(50 \mathrm{eV})$ the dissociation proceeds primarily through the initial charge separation 
mechanism (mechanism 2 above), with a shift to the deferred charge separation mechanism (mechanism 3 above) at higher energies, above $200 \mathrm{eV}{ }^{56,57}$ At even higher energies the concerted mechanism becomes accessible. ${ }^{58}$ At $100 \mathrm{eV}$ we therefore expect to see dynamics corresponding to a mixture of the initial and deferred charge separation mechanisms. Figure 7 shows simulated covariancemap images for both mechanisms together with the experimentally measured images. The simulations were based on a simple energetic model employing fixed energy release values of $1 \mathrm{eV}$ for the $\mathrm{F}$ loss step and $4.5 \mathrm{eV}$ for the charge separation step, based on barrier heights and kinetic energy releases reported by Eland et al. ${ }^{57}$ and Douglas. ${ }^{56}$ In reality there is likely to be a distribution of energy releases in each step. However, we see that the simulated images rather convincingly reproduce the experimentally observed product velocities. As observed previously in Section 3.2.2, the two mechanisms yield very different covariance maps. However, neither pair of simulated images exactly reproduce the features observed in the experimental images. Instead, the experimental images have a form somewhere in between the two pairs of simulated images. This is entirely consistent with the earlier observations on the dissociation dynamics of $\mathrm{CF}_{3} \mathrm{I}^{2+}$. Details of other dissociation channels can be found in reference. ${ }^{33}$

\subsubsection{Dissociation of polycyclic aromatic hydrocarbons: fluorene}

Having demonstrated that recoil-frame covariance-map imaging can be useful in distinguishing between competing dissociation mechanisms in a small molecule, one might wonder whether such an approach can be extended to much larger molecular systems. We have recently investigated the dissociation pathways followed by multiply charged polycyclic aromatic hydrocarbons (PAHs) generated by XUV photoionization, ${ }^{59}$ and have found covariance mapping to be an extremely powerful tool for unravelling the complex dissociation dynamics of these molecules.

As an example, Figure 8(a) shows the time-of-flight mass spectrum recorded for the PAH fluorene $\left(\mathrm{C}_{13} \mathrm{H}_{10}\right)$ following irradiation by $30.3 \mathrm{~nm}$ light from the FLASH X-ray free-electron laser. The spectrum indicates formation of singly, doubly, and triply charged parent ions, along with a wide variety of smaller fragment ions. We will focus in the following on the dissociation dynamics of the dication. The time-of-flight resolution is not sufficient to resolve individual hydrogen atoms unambiguously, so in the following we will group together the signals from $\mathrm{C}_{n} \mathrm{H}_{m}^{+}$ions with different numbers $m$ of hydrogen atoms, and con- 
(a) Initial charge separation

$\mathrm{CF}_{3} \mathrm{I}^{2+} \rightarrow \mathrm{CF}_{3}^{+}+\mathrm{I}^{+}$

$\mathrm{CF}_{3}^{+} \rightarrow \mathrm{CF}_{2}^{+}+\mathrm{F}$

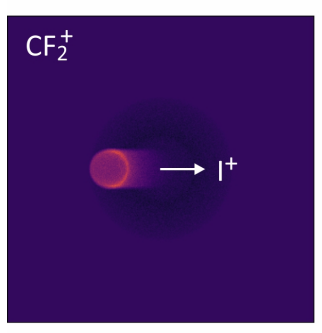

$\mathrm{I}^{+}$

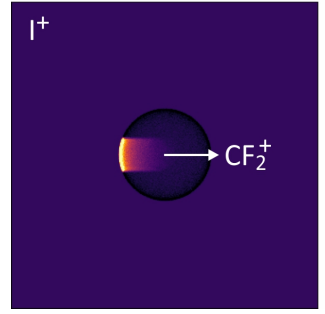

(b) Deferred charge separation (c) Experiment $\mathrm{CF}_{3} \mathrm{I}^{2+} \rightarrow \mathrm{CF}_{2} \mathrm{I}^{2+}+\mathrm{F}$
$\mathrm{CF}_{2} \mathrm{I}^{2+} \rightarrow \mathrm{CF}_{2}^{+}+\mathrm{I}^{+}$ $\mathrm{CF}_{2} \mathrm{I}^{2+} \rightarrow \mathrm{CF}_{2}^{+}+\mathrm{I}^{+}$
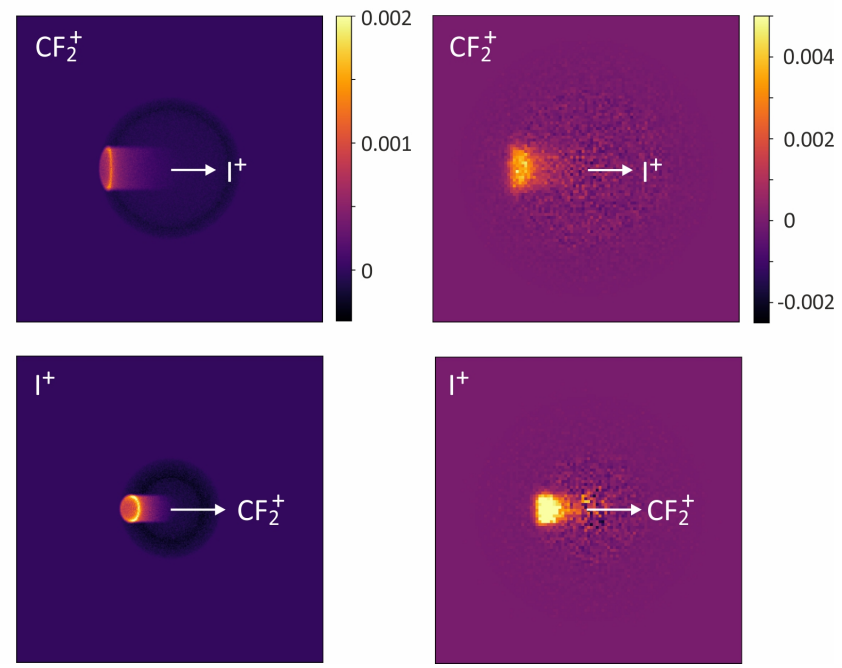

Figure 7: Simulated covariance-map images for dissociation of $\mathrm{CF}_{3} \mathrm{I}^{2+}$ by (a) the initial charge separation mechanism and (b) the deferred charge separation mechanism, together with (c) the experimentally measured images. Reproduced with permission from reference. ${ }^{33}$

sider only the fragmentation dynamics of the carbon framework. We can use covariance mapping to obtain further insight into these dynamics.

As we have seen previously, covariance mapping provides a straightforward and unambiguous way to identify pairs of ions formed in a given process. As an example, Figure 8(b) shows the velocity-map images recorded for the $\mathrm{C}_{2} \mathrm{H}_{m}^{+}$and $\mathrm{C}_{11} \mathrm{H}_{m}^{+}$ions (top row), and the corresponding covariance-map images for the two ions (bottom row). The strong covariance signal reveals that the two fragments are formed via two body dissociation of the doubly-charged parent ion $\mathrm{C}_{13} \mathrm{H}_{10}^{2+}$, and recoil in opposite directions in line with conservation of momentum. We can perform the same analysis for all possible pairs of ions that could be formed from the fluorene dication, and the resulting covariance maps are shown in Figure 9. While there is a great deal of information presented in this figure, it can be distilled down to a relatively simple picture of the fragmentation dynamics.

Firstly, consider the covariance maps appearing along the diagonal in Figure 9. In terms of the carbon framework, these all correspond to two-body dissociation processes of the form $\mathrm{C}_{13}^{2+} \rightarrow \mathrm{C}_{n}^{+}+\mathrm{C}_{13-n}^{+}$. We observe all possible 
(a)
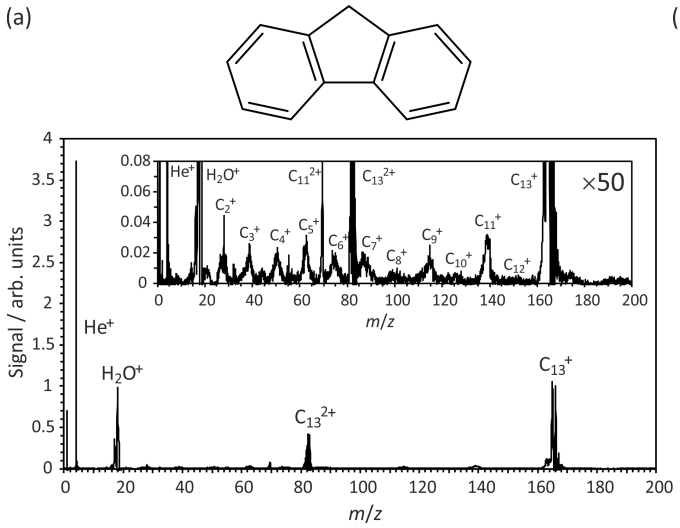

(b)
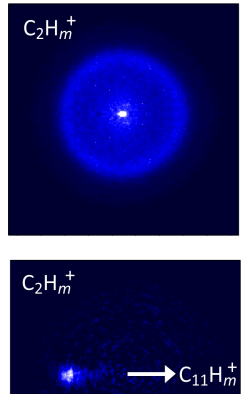

$\mathrm{C}_{11} \mathrm{H}_{m}^{+}$

$*$

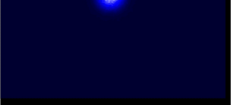

$\mathrm{C}_{11} \mathrm{H}_{m}^{+}$

$\rightarrow \mathrm{C}_{2} \mathrm{H}_{m}^{+}$

Figure 8: (a) time-of-flight spectrum recorded for fluorene (top) following irradiation by $30.3 \mathrm{~nm}$ light from an X-ray free-electron laser; (b) Velocity-map images (top) and recoil-frame covariance-map images (bottom) for the $\mathrm{C}_{2} \mathrm{H}_{m}^{+}$ and $\mathrm{C}_{11} \mathrm{H}_{m}^{+}$fragments.

two-body dissociations of this type apart from loss of a single carbon atom from the parent dication. This is perhaps surprising given the extensive molecular rearrangement and multiple bond cleavages needed to access some of these pathways. However, calculations show that the relevant dissociation energies are all energetically accessible following double ionisation by a $30.3 \mathrm{~nm}$ photon. ${ }^{59}$

On closer inspection of the array of covariance images, we note an interesting pattern in the data. In working down the first column of images, for example, we see the expected covariance between the $\mathrm{C}_{2}^{+}$and $\mathrm{C}_{11}^{+}$fragment ions. However, we also see covariances for the pairs $\left(\mathrm{C}_{2}^{+}, \mathrm{C}_{9}^{+}\right)$and $\left(\mathrm{C}_{2}^{+}, \mathrm{C}_{7}^{+}\right)$. The covariance signals become more blurred as we move from $\mathrm{C}_{11}^{+}$to $\mathrm{C}_{9}^{+}$to $\mathrm{C}_{7}^{+}$. We have seen such effects before, in Section 4.1.1, when we were looking at sequential loss of $\mathrm{F}$ atoms from $\mathrm{CF}_{3} \mathrm{I}^{2+}$ cations. In this case we are not looking at sequential loss of atoms, but of (most probably) neutral acetylene, $\mathrm{C}_{2} \mathrm{H}_{2}$. In terms of the carbon framework, the covariance images reveal the existence of the following sequential dissociation process:

$$
\begin{aligned}
\mathrm{C}_{13}^{2+} & \rightarrow \mathrm{C}_{2}^{+}+\mathrm{C}_{11}^{+} \\
\mathrm{C}_{11}^{+} & \rightarrow \mathrm{C}_{9}^{+}+\mathrm{C}_{2} \\
\mathrm{C}_{9}^{+} & \rightarrow \mathrm{C}_{7}^{+}+\mathrm{C}_{2}
\end{aligned}
$$

In fact, the covariance images reveal that all of the singly-charged ions formed 


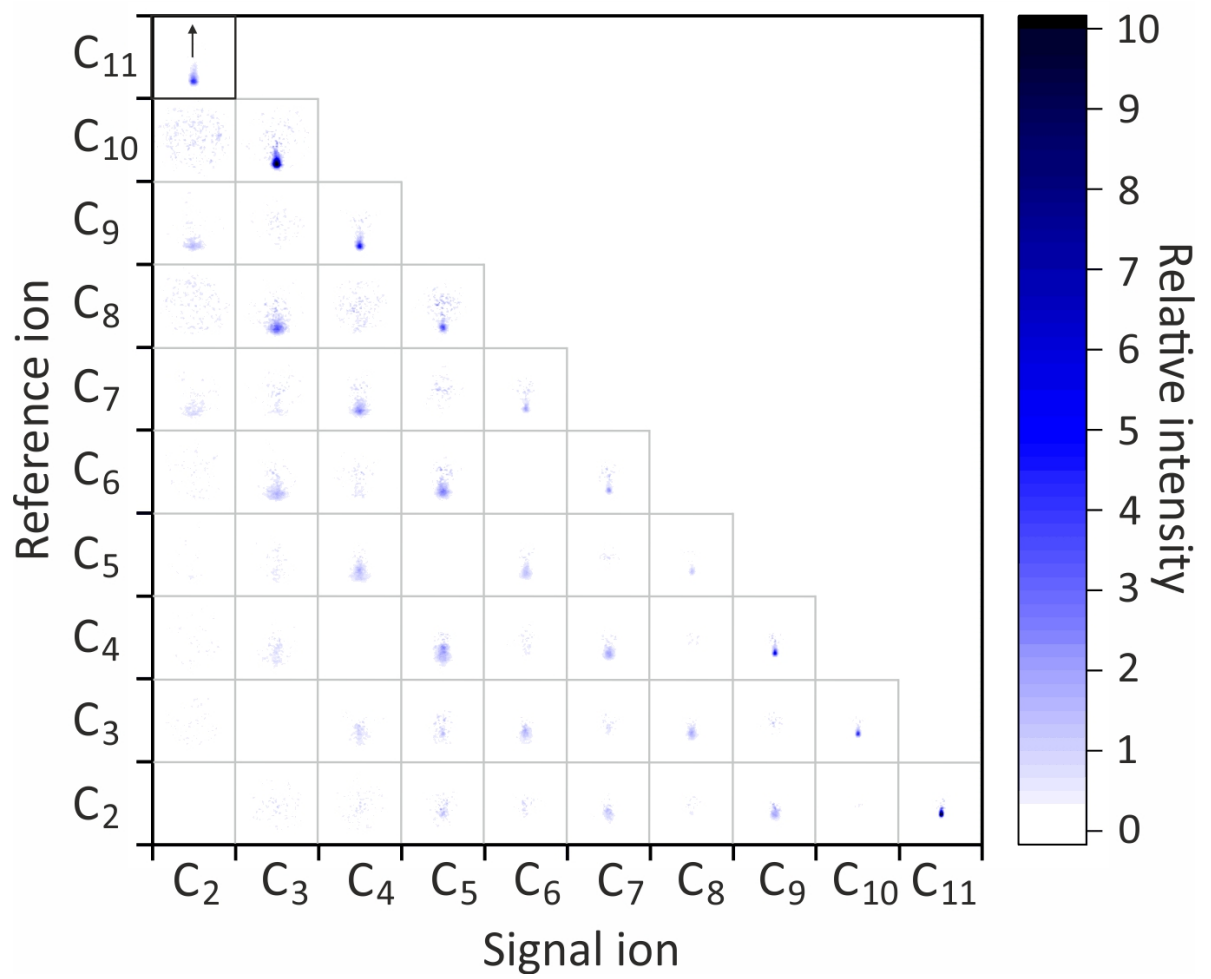

Figure 9: Recoil frame covariance-map images for the various possible ion pairs formed following $30.3 \mathrm{~nm}$ laser ionisation of fluorene. Note that the covariance maps are resolved only by the number of carbon atoms, $\mathrm{C}_{n}$ within each ionic fragment. The reference direction is vertically upwards, as illustrated by the arrow in the covariance-map image of $\mathrm{C}_{2}$ relative to $\mathrm{C}_{11}$ fragments in the top left image of the array.

in the initial dissociation of the dication can decay via the sequential acetylene loss pathway. Working down any of the columns of the images in Figure 9 we see covariances in every second row of the array, which become increasingly blurred as we move down the column. Remarkably, in this particular experiment, a single multi-mass imaging data set combined with covariance analysis of the data allows us to observe an entire network of competing multi-step dissociation pathways. These pathways are summarised in Figure 10. 


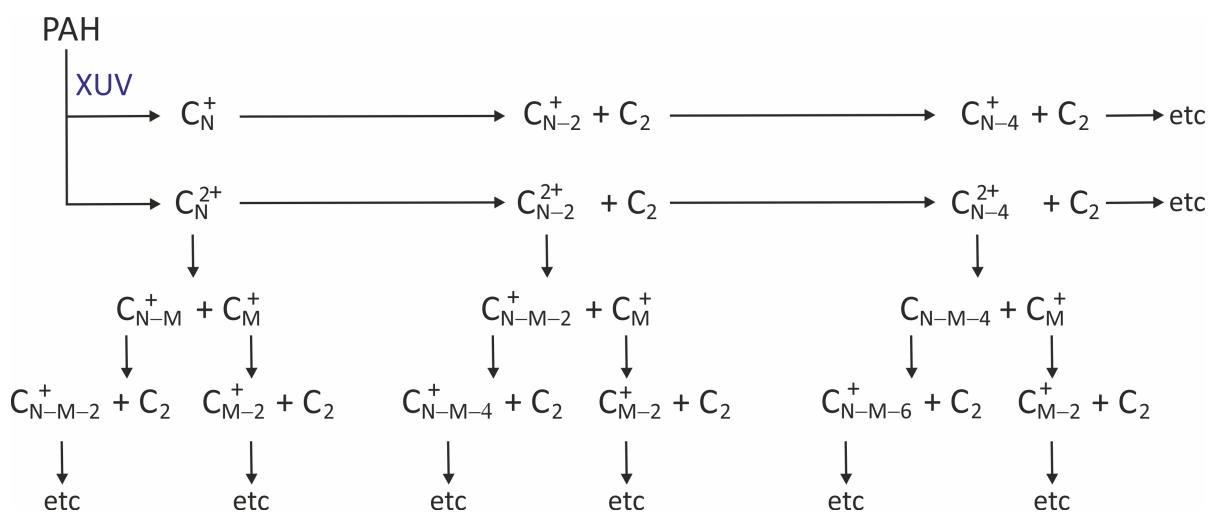

Figure 10: Fragmentation pathways of the carbon framework in fluorene, as determined from covariance-map imaging. Singly-charged PAH cations decay via sequential loss of neutral $\mathrm{C}_{2} \mathrm{H}_{x}$ fragments. Doubly-charged PAHs also decay via this pathway, or alternatively can undergo a pairwise dissociation into two smaller singly-charged ions, which then decay further via sequential loss of $\mathrm{C}_{2} \mathrm{H}_{x}$.

\subsection{Molecular structure determination: covariance analy- sis of Coulomb explosion images}

We have shown that covariance-map imaging is proving to be a powerful tool for probing a wide variety of photon and electron-induced dynamics. However, it is also capable of providing direct information on molecular structures, and in some cases even enables the recording of 'molecular movies': real time visualisations of chemical dynamics.

To obtain structural information, covariance mapping is used in combination with Coulomb explosion imaging. A focused ultrafast laser pulse is sufficiently powerful to strip multiple valence electrons from a molecule, removing some or all of the chemical bonds. This can be achieved either directly via strong-field ionization, or indirectly by core electron ionisation followed by an Auger cascade, with similar ensuing dynamics in the two cases. ${ }^{60}$ If the laser pulse is sufficiently short that neglible nuclear motion occurs during the stripping process, the result is a set of positively charged atoms or small molecular fragments located at the original positions as the neutral atoms in the original molecule. Strong Coulomb repulsion between the charges then rapidly leads to a Coulomb explosion, during which the initial positions of the fragments are mapped onto their final velocities. Multi-mass velocity-map imaging allows the velocities of all fragments to be recorded, which in principle allows one to 'work backwards' 
to the original structure. A caveat is that the mapping from initial position to final velocity is only straightforward if sufficiently high charge states of the parent ion can be accessed. Low charge states tend to exhibit complex dissociation dynamics, in which attractive intermolecular forces play an important role $^{61-66}$ and the final trajectories depend on a variety of factors, including the charge state, vibrational state, and electronic state of the ion. In contrast, for high charge states the dynamics are dominated by Coulomb repulsion and are relatively insensitive to other factors. We have recently investigated these effects in detail by means of a simulation study on multiple charge states of formyl chloride and cis-1,2-dichloroethene. ${ }^{65}$ The origins of non-axial recoil in Coulomb explosions have also recently been studied experimentally. ${ }^{67,68}$

Coulomb explosions of small molecules have been studied by coincidence imaging for several decades (see Section 2). Under coincidence conditions the Coulomb explosion products are detected for one exploding molecule at a time, and the velocities can be rotated into a common reference frame in order to uncover the relationships between the velocities of the different fragments. A very good overview of the requirements for such experiments has been provided by Pitzer et al. ${ }^{69}$ Such experiments have focused almost exclusively on understanding the Coulomb explosion dynamics of small molecules for which the molecular structures are already well established, and while in principle they could be extended to larger molecules, insufficiently high detection sensitivity is likely to present significant challenges (see Section 3.3.2). In contrast, multi-mass velocity-map imaging experiments can record images for all of the fragmentation products, though in general not every fragment will be detected from each parent ion. At the typical count rates used in such experiments, the products of multiple Coulomb explosions are recorded on each experimental cycle. This has the result that within the velocity-map images, the superposition of multiple trajectories arising from parent molecules with many initial orientations obscures the relationships between the fragment velocities. Covariance analysis provides a means to recover this information.

As an example, we will consider a series of experiments carried out by Stapelfeldt and coworkers ${ }^{36,37,70}$ to probe Coulomb explosion of the substituted biphenyl molecule 3,5-dibromo- 3',5'-difluoro-4'-cyanobiphenyl, shown in Figure 11(a). To simplify interpretation of the velocity-map images, the molecule was prealigned with respect to a laboratory axis using a linearly polarised nanosecond pulse from a Nd:YAG laser. ${ }^{71-77}$ This aligns the molecular axis to the polarisation axis of the laser beam, but the molecule still undergoes free rotation 
(a)

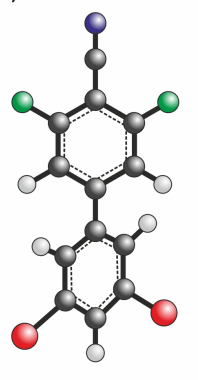

(b) $\quad \mathrm{H}^{+} \quad \mathrm{C}^{2+} \quad \mathrm{N}^{2+} \quad \mathrm{F}^{2+} \quad \mathrm{C}^{+} \quad \mathrm{N}^{+} \quad \mathrm{F}^{+} \quad \mathrm{C}_{2}^{+} \mathrm{CN}^{+} / \mathrm{Br}^{3+} \mathrm{Br}^{2+} \quad \mathrm{Br}^{+}$

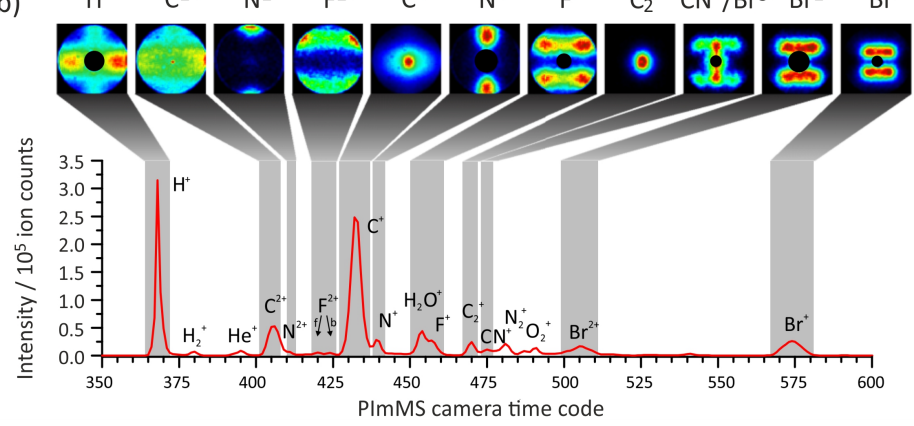

Figure 11: (a) Molecular structure of the substituted biphenyl molecule used in the experiments described in the text; (b) time-of-flight spectrum and velocitymap images recorded for the Coulomb-explosion products of the substituted biphenyl molecule in (a). Adapted from reference ${ }^{37}$.

about this axis. It is also important to note that the molecule is only aligned, not oriented, so half of the molecules in the sample are oriented as shown in Figure 11(a), and the other half are 'upside down'. The aligned molecules were subjected to a high intensity $\sim 30 \mathrm{fs}$ pulse from a Ti:Sapphire laser to initiate Coulomb explosion, and the resulting ions were recorded in a multi-mass velocity-map imaging measurement employing the PImMS camera. Figure 11(b) shows the mass spectrum and velocity-map images for each detected fragment, as extracted directly from the PImMS data set. Note that the alignment axis lies vertically in the images. The mass spectrum shows peaks for all of the expected singly and doubly charged atomic fragment ions $\left(\mathrm{H}^{+}, \mathrm{C}^{+}, \mathrm{C}^{2+}, \mathrm{F}^{+}\right.$, $\left.\mathrm{F}^{2+}, \mathrm{N}^{+}, \mathrm{Br}^{+}, \mathrm{Br}^{2+}\right)$ and small molecular fragment ions $\left(\mathrm{C}_{2}^{+}\right.$and $\left.\mathrm{CN}^{+}\right)$. Even the 'raw' velocity-map images contain a significant amount of structural information, though this is averaged over the various orientations of the sample molecules. For example, based on the presence of a cyano (CN) group at the 'top' of the molecule, we expect to see $\mathrm{N}^{+}$ions to be formed flying upwards for the molecules oriented as shown in Figure 11(a), and flying 'downwards' for those oriented 'upside down'. The $\mathrm{N}^{+}$image does indeed reveal $\mathrm{N}^{+}$ions travelling in both the upwards and downwards directions relative to the alignment axis. We can make similar arguments for the other atomic fragments.

Figure 12 shows covariance-map images constructed from several pairs of the images shown in Figure 11(b). In each image, $\mathrm{N}^{+}$has been chosen as the reference ion, and the three images reveal the velocity distributions of the $\mathrm{F}^{+}$, $\mathrm{Br}^{+}$and $\mathrm{H}^{+}$ions relative to this direction. The velocity distributions clearly 

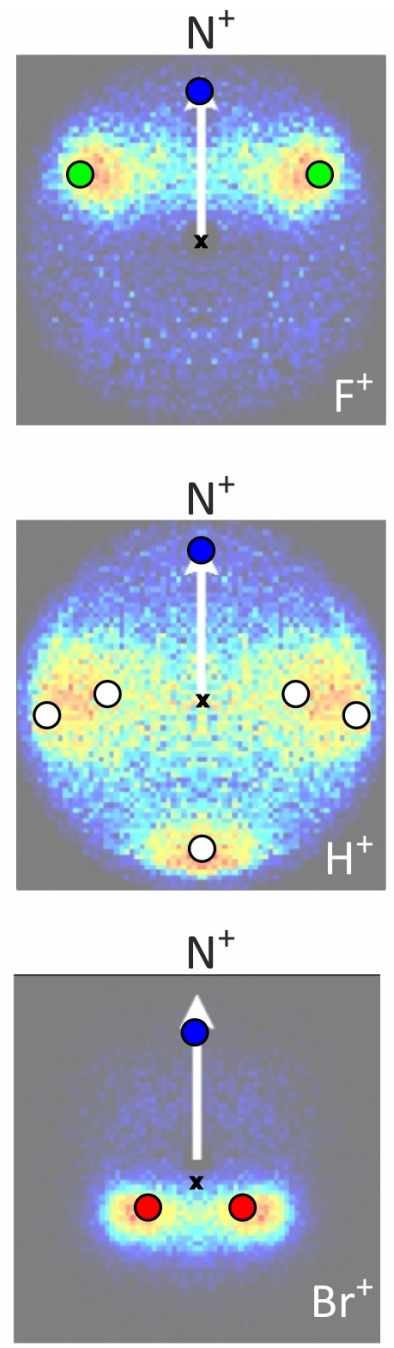

Figure 12: Covariance-map images for various ion pairs formed in the Coulomb explosion of the substituted biphenyl molecule shown in Figure 11(a). In each image, $\mathrm{N}^{+}$is the reference ion, and travels in the upwards direction in each image. The circles superimposed on each image are the result of trajectory simulations based on a simple model described in the text. 
reflect the spatial relationships between the corresponding atoms in the original biphenyl molecule, though we note that because of the $2 \mathrm{D}$ projection involved in the velocity-mapping measurement, the covariance calculation does not correct for the distribution of orientations caused by rotation about the molecular axis, and this 'smears out' the measured velocity distributions somewhat. This could be addressed by performing a 3D covariance measurement of the type described in Section 3.3.3.

The circles superimposed on each image show the results of a very simple trajectory simulation which assigns a single charge to each atom in the parent molecule, and solves Newton's equations assuming that Coulomb repulsion is the only force acting on the ions. Much more sophisticated simulations are reported in reference, ${ }^{37}$ but we see that even this simple model is able to predict the measured fragment velocities with a reasonable degree of accuracy. An eventual goal is to develop a structure refinement procedure analogous to that used in $\mathrm{X}$-ray crystallography, in which an 'initial guess' structure is refined iteratively to optimise the agreement between the predicted and measured covariance-map images.

The fact that laser-induced Coulomb explosions are initiated on the femtosecond timescale offers the intriguing possibility of probing molecular structure in real time over the course of a chemical change. In addition to 'static' Coulomb explosion measurements, the substituted biphenyl described above has also been the subject of time-resolved measurements. ${ }^{70,78-80}$ For these measurements the molecules were aligned with their molecular axis pointing along the time-of-flight axis, i.e. perpendicular to the image plane. Following alignment of the molecular axis by the nanosecond laser pulse, the aligned molecules were subjected to a pair of femtosecond laser pulses with a variable delay between the two pulses. The first pulse initiated a vibrational mode involving a torsional motion of the two phenyl rings, resulting in an oscillation of the dihedral angle between the two rings about its equilibrium value of close to 45 degrees. The second pulse initiated Coulomb explosion. By varying the delay between the two pulses, and recording velocity-map images of the Coulomb explosion products as described above, the molecular structure can be investigated at various different times during the vibration, either directly from the velocity-map images, ${ }^{80}$ or more directly from the covariance-map images. ${ }^{70}$ Figure $13(\mathrm{a})$ shows the dihedral angle recovered from the covariance-map images plotted as a function of time. We see a clear oscillation with an amplitude of 3 degrees and a period of 1.22 ps. As shown in Figures 13(b) and (c), the authors also showed that it 

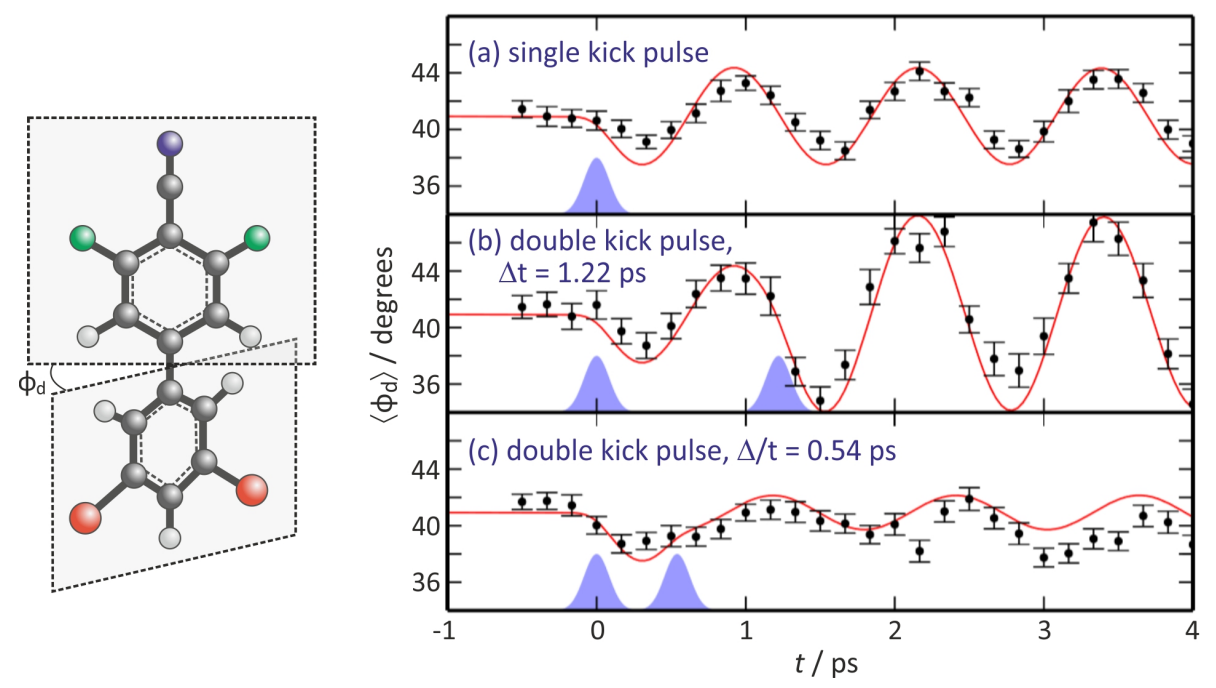

Figure 13: Dihedral angle between the two phenyl rings of 3,5-dibromo- 3',5'difluoro-4'-cyanobiphenyl plotted as a function of time after one or two kick pulses (blue Gaussians) that initiate a torsional vibration. The dihedral angle is determined from the covariance images of the $\left(\mathrm{F}^{+}, \mathrm{Br}^{+}\right)$ion pair when the Coulomb explosion fragments are imaged for molecules aligned 'end on' to the detector. Traces are shown for (a) a single kick pulse; (b) two kick pulses separated by the vibrational period of $1.22 \mathrm{ps}$; (c) two kick pulses separated by $0.54 \mathrm{ps}$, slightly less than half a vibrational period. Experimental results are shown as black dots; red lines show calculated results. Adapted from reference. ${ }^{70}$ 
is possible to double the amplitude of the vibration by including an additional laser pulse a full vibrational cycle after the first kick pulse, and to quench the vibrational motion via destructive interference by including an additional laser pulse close to half a cycle (0.54 ps) after the first.

There have been a number of other recent examples of covariance mapping applied to the products of Coulomb explosions. For example, Burt et $a l .{ }^{39}$ showed that the approach could be used to distinguish between structural isomers of difluoroiodobenzene and dihydroxybromobenzene in the gas phase. Several of the same authors have also shown that three-fold covariance mapping (see Section 3.3.2) applied to the products of Coulomb explosions can be used to establish the absolute configuration of a chiral molecule. ${ }^{51}$ Stapelfeldt and coworkers have used Coulomb explosion imaging combined with covariance mapping to investigate both the degree of alignment ${ }^{68,81-83}$ and the detailed structures of individual molecules and small molecular clusters contained within helium nanodroplets. ${ }^{84,85}$

\section{Future directions}

Covariance-mapping applied to multi-mass velocity-map images is a technique still in its infancy, and we continue to discover new ways in which this versatile approach can help us to probe such data sets in ever more detail. We anticipate that the technique will be used increasingly to isolate signal contributions from individual reaction pathways in systems possessing multiple competing pathways. The ability of covariance mapping to identify stepwise reaction pathways, as shown in Sections 4.1.1 and 4.1.2, is likely to prove particularly useful.

The use of covariance mapping in combination with Coulomb explosion imaging shows significant promise as an ultra-fast molecular structure determination method. However, there is a great deal of work still to do in understanding the capabilities and limitations of the approach. In particular, while the method has proven to be very effective in tracking bond angles, the extent to which accurate bond lengths can be recovered has not yet been established with any degree of rigour. In addition, most demonstrations of the approach to date have focused on relatively rigid molecules containing one or more heavy atoms which are exploited as 'mass tags' during the data analysis. Systematic studies will need to be performed under a variety of experimental conditions on a series of 'benchmark' molecules in order to explore the extent to which the approach is 
generally applicable, as well as to determine the precision and accuracy that can be achieved in structure measurements.

Both mechanistic and structural studies are likely to benefit over the coming years from continued improvements in experimental capabilities. In parallel with advances in data analysis capabilities, improved universal ionisation schemes coupled with faster and more sensitive detection systems will enhance covariance signals and reduce false covariances within measured data sets. Faster detectors will also open the way to making ' $3 \mathrm{D}$-sliced' velocity-map imaging measurements routine, enabling covariances to be observed in three dimensions rather than two. This is likely to prove particularly useful in structure determination applications for extracting full 3D structural information from a data set, and will also simplify interpretation of covariance maps in mechanistic studies.

While this review has focused on covariances between ions, there is also considerable scope for covariance mapping to be used in the study of ionisation processes. Photoelectron imaging was first demonstrated during the relatively early days of ion imaging, ${ }^{86,87}$ before the discovery of velocity-map imaging, and the higher-resolution method of velocity-map imaging is now widely used to study such processes, ${ }^{88-90}$ and has provided rich insight into the electronic states involved. A variety of photoelectron-photoion coincidence methods are also widely employed (see Section 2). Covariance analysis can provide similar information to that available from a coincidence experiment, but allows experiments to be performed under a wider range of conditions, and in particular at higher count rates. This opens up a range of possibilities in terms of the types of light source that can be used. In the majority of photoelectron-photoion coincidence experiments, the electron and ions are recorded at separate detectors. Applying covariance analysis in such situations requires careful co-registration of signals to ensure that the electron and ion signals from each experimental cycle can be analysed together. For example, Rading et al. have recently described a purpose-built experiment of this type ${ }^{91}$ Alternatively, recording both electrons and positive ions on a single detector in a multi-mass velocity-map imaging experiment has already been demonstrated, ${ }^{92,93}$ and requires only the replacement of static velocity-map imaging lens potentials with potentials that switch from negative to positive in order to extract electrons and ions from the interaction region in sequence.

In summary, while there is much work still to be done before covariance-map imaging becomes a fully mature technology, we believe that the approach has huge potential in extending chemical dynamics studies to much larger chemical 
systems than have been investigated to date. We anticipate that over the coming decades the technique will provide deep insight into a wide range of chemical systems of real-world interest, spanning atmospheric chemistry, astrochemistry, chemical biology, synthetic photochemistry, photomedicine, and more.

\section{Acknowledgements}

We would like to thank the very large number of collaborators who we have worked with on the studies described in this review. Particular thanks goes to the group leaders involved in these studies - Mark Brouard, Melanie Schnell, and Henrik Stapelfeldt, as well as to Richard Chapman, Gabriel Karras, and Emma Springate at the Artemis ultrafast laser facility, the members of the PImMS collaboration (https://pimms.chem.ox.ac.uk) and the technical teams at both Artemis and the FLASH X-ray free electron laser facility. This work was supported by the EPSRC under Programme Grants EP/L005913/1 and

EP/T021675/1 and a Doctoral Training Grant to DH, and through access to beamtime at Artemis and FLASH.

\section{Disclosure statement}

The authors have no conflicts of interest to declare.

\section{Data availability statement}

The data files are available on request by contacting the corresponding author.

\section{References}

[1] Eppink, A. T. J. B.; Parker, D. H. Velocity map imaging of ions and electrons using electrostatic lenses: Application in photoelectron and photofragment ion imaging of molecular oxygen. Rev. Sci. Instrum. 1997, 68,3477 .

[2] Chandler, D. W.; Houston, P. L. Two-dimensional imaging of state-selected photodissociation products detected by multiphoton ionization. J. Chem. Phys. 1987, 87, 1445. 
[3] B. J. Whitaker, E. Imaging in Molecular Dynamics; Cambridge University Press, 2003.

[4] Suits, A. G.; R. E. Continetti, E. Imaging in Chemical Dynamics; American Chemical Society, 2001.

[5] Ashfold, M. N. R.; Parker, D. H. Imaging molecular dynamics. Phys. Chem. Chem. Phys. 2014, 16, 381, Special themed collection on Imaging Molecular Dynamics.

[6] Chandler, D. W.; Houston, P. L.; Parker, D. H. Perpsective: Advanced Particle Imaging. J. Chem. Phys. 2017, 147, 013601.

[7] Townsend, D.; Lahankar, S. A.; Lee, S. K.; Chambreau, S. D.; Suits, A. G.; Zhang, X.; Rheinecker, J.; Harding, L. B.; Bowman, J. M. The roaming atom: straying from the reaction path in formaldehyde decomposition. Science 2004, 306, 1158.

[8] Hickstein, D. D.; Yurchak, R.; Das, D.; Shih, C. Y.; Gibson, S. T. PyAbel: a Python package for Abel transforms. 2018.

[9] Gebhardt, C. R.; Rakitzis, T. P.; Samartzis, P. C.; Ladopoulos, V.; Kitsopoulos, T. N. Slice imaging: a new approach to ion imaging and velocity mapping. Rev. Sci. Instrum. 2001, 72, 3848.

[10] Dinu, L.; Eppink, A. T. J. B.; Rosca-Pruna, F.; Offerhaus, H. L.; van der Zande, W. J.; Vrakking, M. J. J. Application of a time-resolved event counting technique in velocity-map imaging. Rev. Sci. Instrum. 2002, 73, 4206.

[11] Townsend, D.; Minitti, M. P.; Suits, A. G. Direct current slice imaging. Rev. Sci. Instrum. 2003, 74, 2350.

[12] Lin, J. J.; Zhou, J.; Shiu, W.; Liu, K. Applicatoin of time-sliced ion velocity imaging to crossed molecular beam experiments. Rev. Sci. Instrum. 2003, 74, 2495.

[13] Ashfold, M. N. R.; King, G. A.; Murdock, D.; Nix, M. G. D.; Oliver, T. A. A.; Sage, A. G. $\pi \sigma *$ excited states in molecular photochemistry. Phys. Chem. Chem. Phys. 2010, 12, 1218.

[14] Roberts, G. M.; Williams, C. A.; Young, J. D.; Ullrich, S.; Paterson, M. J.; Stavros, V. G. Unraveling ultrafast dynamics in photoexcited aniline. $J$. Am. Chem. Soc. 2012, 134, 12578. 
[15] Roberts, G. M.; Chatterley, A. S.; Young, J. D.; Stavros, V. G. Direct observation of hydrogen tunneling dynamics in photoexcited phenol. J. Phys. Chem. Lett. 2012, 3, 348.

[16] Roberts, G. M.; Hadden, D. J.; Bergendahl, L. T.; Wenge, A. M.; Harris, S. J.; Karsili, T. N. V.; Ashfold, M. N. R.; Paterson, M. J.; Stavros, V. G. Exploring quantum phenomena and vibrational control in $\sigma *$ mediated photochemistry. Chem. Sci. 2013, 4, 993.

[17] Blackshaw, K. J.; Marracci, M.; Korb, R. T.; Quartey, N.-K.; Ajmani, A. K.; Hood, D. J.; Abelt, C. J.; Ortega, B. I.; Luong, K.; Petit, A. S. et al. Dynamical signatures from competing nonadiabatic fragmentation pathways of S-nitrosophenol. Phys. Chem. Chem. Phys. 2020, 22, 12187.

[18] Zhang, J.; Li, G.; Yuan, Q.; Zou, J.; Yang, D.; Cheng, H.; Wang, C.; Yang, J.; Jing, Q.; Liu, Y. et al. Photelectron velocity map iamging spectroscopic and theoretical study of heteronuclear $\mathrm{NMi}(\mathrm{CO})_{7}^{-}(\mathrm{M}=\mathrm{V}, \mathrm{Nb}$, Ta). J. Phys. Chem. A 2020, 124, 2264.

[19] Ahmed, M.; Kostco, O. From atoms to aerosols: probing clusters and nanoparticles with synchrotron based mass spectrometry and X-ray spectroscopy. Phys. Chem. Chem. Phys. 2020, 22, 2713.

[20] Vallance, C. Multi-mass velocity-map imaging of photoinduced and electron-induced chemistry. Chem. Comm. 2019, 55, 6336.

[21] Brehm, B.; von Puttkammer, E. Koinzidensmessung von Photoionen und Photoelektronen bei Methan. Z. Naturforsch. A 1967, 22, 8.

[22] Continetti, R. E.; Hayden, C. C. Coincidence imaging techniques. Advanced Series in Physical Chemistry: Modern Trends in Chemical Reaction Dynamics 2004, 14, 475 .

[23] Vager, Z. Coulomb explosion imaging of molecules. Adv. At. Mol. Opt. Phys. 2001, 45, 203.

[24] Bocharova, I. Laser Coulomb explosion imaging of molecular dynamics; Lampert Academic Publishing, 2011.

[25] R. Karimi, W.-K. L.; Sanderson, J. Femtosecond laser-induced Coulomb explosion imaging. Advances in Multi-Photon Processes and Spectroscopy 2016, 23, 97. 
[26] Légaré, F.; Lee, K. F.; Bandrauk, A. D.; Villeneuve, D. M.; Corkum, P. B. Laser Coulomb explosion imaging for probing ultra-fast molecular dynamics. J. Phys. B: At. Mol. Opt. Phys. 2006, 39, S503.

[27] Hishikawa, A.; Yamanouchi, K. Progress in ultrafast laser science II. Springer Series in Chemical Physics, vol. 85; Springer: Berlin, Heidelberg, 2007; Vol. 85; Chapter 1, pp 1-24.

[28] Mikosch, J.; Patchkovskii, S. Coincidence and covariance data acquisition in photoelectron and ion spectroscopy. II. Analysis and applications. $J$. Mod. Opt. 2013, 60, 1439.

[29] Frasinski, L. J.; Codling, K.; Hatherly, P. A. Covariance mapping: a correlation method applied to multiphoton multiple ionization. Science $\mathbf{1 9 8 9}$, $246,1029$.

[30] Mikosch, J.; Patchkovskii, S. Coincidence and covariance data acquisition in photoelectron and ion spectroscopy. I. Formal theory. J. Mod. Opt. 2013, 60, 1426.

[31] Bhardwaj, V. R.; Vijayalakshmi, K.; Mathur, D. Dissociative ionization of benzene in intense laser fields of picosecond duration. Phys. Rev. A 1999, 59, 1392.

[32] Frasinski, L. J. Covariance mapping techniques. J. Phys. B 2016, 49, 152004.

[33] Köckert, H.; Heathcote, D.; Lee, J. W. L.; Vallance, C. Covariance-map imaging study into the fragmentation dynamics of multiply-charged $\mathrm{CF}_{3} \mathrm{I}$ formed in electron-molecule collisions. Mol. Phys. 2020, published online September 2020.

[34] Eland, J. H. D.; Wort, F. S.; Royds, R. N. A photoelectron-ion-ion triple coincidence technique for the study of double photoionization and its consequences. J. Electron Spectr. Rel. Phenom. 1986, 41, 297.

[35] Eland, J. H. D. The dynamics of three-body dissociations of dications studied by the triple coincidence technique PEPIPICO. Mol. Phys. 1987, 61, 725 . 
[36] Slater, C. S.; Blake, S.; Brouard, M.; Lauer, A.; Vallance, C.; John, J. J.; Turchetta, R.; Nomerotski, A.; Christensen, L.; Nielsen, J. H. et al. Covariance imaging experiments using the Pixel Imaging Mass Spectrometry camera. Phys. Rev. A 2014, 89, 011401(R).

[37] Slater, C. S.; Blake, S.; Brouard, M.; Lauer, A.; Vallance, C.; Bohun, S.; Christensen, L.; Nielsen, J. H.; Johansson, M. P.; Stapelfeldt, H. Coulomb explosion imaging using a Pixel Imaging Mass Spectrometry camera. Phys. Rev. A 2015, 91, 053424.

[38] Bargheer, M.; Zhavoronkov, N.; Woerner, M.; Elsaesser, T. Recent progress in ultrafast X-ray diffraction. ChemPhysChem 2006, 7, 783.

[39] Burt, M.; Amini, K.; Lee, J. W. L.; Christiansen, L.; Johansen, R. R.; Kobayashi, Y.; Pickering, J. D.; Vallance, C.; Brouard, M.; Stapelfeldt, H. Gas-phase structural isomer identification by Coulomb explosion of aligned molecules. J. Chem. Phys. 2018, 148, 091102.

[40] Amini, K.; Boll, R.; Lauer, A.; Burt, M.; Lee, J. W. L.; Christensen, L.; Brausse, F.; Mullins, T.; Savelyev, E.; Abttkim, U. et al. Alignment, orientation, and Coulomb explosion of difluoroiodobenzene studied with the pixel imaging mass spectrometry (PImMS) camera. J. Chem. Phys. 2017, $147,013933$.

[41] Frasinski, L. J. On the absence of negative correlations between products of competing processes. J. Phys. B. 1993, 26, 2225.

[42] Card, D. A.; Folmer, D. E.; Sato, S.; Buzza, S. A.; Jr., A. W. C. Covariance mapping of ammonia clusters: evidence of the connectiveness of clusters with coulombic explosion. J. Phys. Chem. A 1997, 101, 3417.

[43] Card, D. A.; Wisniewski, E. S.; Folmer, D. E.; Jr., A. W. C. The relationship between covariance and anti-covariance mapping. Int. J. Mass Spectrom. 2003, 223-224, 355.

[44] Amoruso, S.; Berardi, V.; Spinelli, N.; adn M. Armenante adn F. Fuso, R. V.; Allegrini, M.; Arimondo, E. Time of flight mass spectrometry adn covariance mapping technique investigation of charged specie evolution in $\mathrm{Pb}\left(\mathrm{Ti}_{0.48} \mathrm{Zr}_{0.52}\right) \mathrm{O}_{3}$ laser ablation. Appl. Surf. Sci. 1995, 86, 35 . 
[45] Berardi, V.; Spinelli, N.; Velotta, R.; Armenante, M.; Fuso, F.; Allegrini, M.; Arimondo, E. Correlation analysis of laser ablated ions from YBCO. Phys. Lett. A 1993, 179, 116.

[46] Krzanowski, W. J. Principles of multivariate analysis: a user's perspective; Oxford University Press, Oxford, 2000.

[47] Frasinski, L. J.; Giles, A. J.; Hatherly, P. A.; Posthumus, J. H.; Thompson, M. R.; Codling, K. Covariance mapping and triple coincidence techniques applied to multielectron dissociative ionization. J. Elec. Spectr. Rel. Phenom. 1996, 79, 367-371.

[48] Zhaunerchyk, V.; Frasinski, L. J.; Eland, J. H.; Feifel, R. Theory and simulations of covariance mapping in multiple dimensions for data analysis in high-event-rate experiments. Phys. Rev. A 2014, 89, 053418.

[49] Frasinski, L. J.; Hatherly, P. A.; Codling, K. Multiphoton multiple ionisation of $\mathrm{N}_{2} \mathrm{O}$ probed by three-dimensional covariance mapping. Phys. Lett. A 1991, 156, 227.

[50] Bryan, W. A.; Newell, W. R.; Sanderson, J. H.; Langley, A. J. Observation of multiple ionization pathways for OCS in an intense laser field resolved by three-dimensional covariance mapping and visualised by hierarchical ionization topology. Phys. Rev. A 2006, 74, 053409.

[51] Christensen, L.; Nielsen, J. H.; Slater, C. S.; Lauer, A.; Brouard, M.; Stapelfeldt, H. Using laser-induced Coulomb explosion of aligned chiral molecules to determine their absolute configuration. Phys. Rev. A 2015, 92, 033411.

[52] Pickering, J. D.; Amini, K.; Brouard, M.; Burt, M.; Bush, I. J.; Christensen, L.; Lauer, A.; Nielsen, J. H.; Slater, C. S.; Stapelfeldt, H. Communication: Three-fold covariance imaging of laser-induced Coulomb explosions. J. Chem. Phys. 2016, 144, 161105.

[53] Pitzer, M.; Kunitski, M.; Johnson, A. S.; Jahnke, T.; Sann, H.; Sturm, F.; Schmidt, L. P. H.; Schmidt-Böcking, H.; Dörner, R.; Stohner, J. et al. Direct determination of absolute molecular stereochemistry in gas phase by Coulomb explosion imaging. Science 2013, 341, 1096. 
[54] Lee, J. W. L.; Köckert, H.; Heathcote, D.; Popat, D.; Chapman, R. T.; Karras, G.; Majchrzak, P.; Springate, E.; Vallance, C. Three-dimensional covariance-map imaging as a probe of molecular structure and dynamics on the ultrafast timescale. Comm. Chem. 2020, 3, 72 .

[55] Köckert, H.; Heathcote, D.; Lee, J. W. L.; Zhou, W.; Richardson, V.; Vallance, C. C-I and C-F bond-breaking dynamics in the dissociative electron ionization of $\mathrm{CF}_{3}$ I. Phys. Chem. Chem. Phys. 2019, 21, 14296.

[56] Douglas, K. M. The formation, dissociation and reactivity of gaseous ions. Ph.D. thesis, University College London, 2011.

[57] Eland, J. H. D.; Feifel, R.; Hochlaf, M. Double photoionization and dication fragmentation of $\mathrm{CF}_{3} \mathrm{I}$ : experiment and theory. J. Chem. Phys. 2008, 128, 234303.

[58] Yan, S.; Ma, X.; Zhu, X. L. Fragmentation of $\mathrm{CF}_{3} \mathrm{I}$ induced by electron collision. 2019.

[59] Lee, J. W. L.; Tikhonov, D. S.; Chopra, P.; Maclot, S.; Steber, A. L.; Gruet, S.; Allum, F.; Boll, R.; Cheng, X.; Erk, B. et al. Time-resolved relaxation and fragmentation of polycyclic aromatic hydrocarbons investigated in the ultrafast XUV-IR regime. In preparation 2020,

[60] Allum, F.; Burt, M.; Amini, K.; Boll, R.; Köckert, H.; Olshin, P. K.; Bari, S.; Bomme, C.; Brausse, F.; de Miranda, B. C. et al. Coulomb explosion imaging of $\mathrm{CH}_{3} \mathrm{I}$ and $\mathrm{CH}_{3} \mathrm{ClI}$ photodissociation dynamics. J. Chem. Phys. 2018, 149, 204313.

[61] Corrales, M. E.; Gitzinger, G.; González-Vásquez, J.; Loriot, V.; de Nalda, R.; anares, L. B. Velocity map imaging and theoretical study of the Coulomb explosion of $\mathrm{CH}_{3} \mathrm{I}$ under intense femtosecond IR pulses. $J$. Phys. Chem. A 2012, 116, 2699.

[62] Liu, H.; Yang, Z.; Gao, Z.; Tang, Z. Ionization and dissociation of $\mathrm{CH}_{3} \mathrm{I}$ in intense laser field. J. Chem. Phys. 2007, 126, 044316.

[63] Zuo, T.; Bandrauk, A. D. Charge-resonance-enhanced ionization of diatomic molecular ions by intense lasers. Phys. Rev. A 1995, 52, R2511. 
[64] Rose-Petruck, C.; Schafer, K. J.; Wildon, K. R.; Barty, C. P. J. Ultrafast electron dynamics and inner-shell ionization in laser driven clusters. Phys. Rev. A 1997, 55, 1182.

[65] Zhou, W.; Ge, L.; Cooper, G.; Crane, S.; Evans, M.; Ashfold, M.; Vallance, C. Coulomb explosion imaging for gas-phase molecular structure determination: an ab initio trajectory simulation study. J. Chem. Phys. 2020, 153, 184201.

[66] Hu, X.; Peng, Y.; Zhu, X.; Yan, S.; Liu, L.; Feng, W.; Guo, D.; Gao, Y.; Zhang, S.; Zhao, D. et al. Breakdown of the Coulomb-explosion imaging technique induced by the ultrafast rotation of fragments. Phys. Rev. A 2020, 101, 012707.

[67] Christensen, L.; Christiansen, L.; Shepperson, B.; Stapelfeldt, H. Deconvoluting nonaxial recoil in Coulomb explosion measurements of molecular axis alignment. Phys. Rev. A 2016, 94, 023410.

[68] Christiansen, L.; Nielsen, J. H.; Christensen, L.; Shepperson, B.; Pentlehner, D.; Stapelfeldt, H. Laser-induced Coulomb explosion of 1,4diiodobenzene molecules: studies of isolated molecules and molecules in helium nanodroplets. Phys. Rev. A 2016, 93, 0233411.

[69] Pitzer, M.; Fehre, K.; Kunitski, M.; Jahnke, T.; Schmidt, L.; SchmidtBöcking, H.; Schöffler, R. D. M. S. Coulomb explosion imaging as a tool to distinguish between stereoisomers. J. Vis. Exp. 2017, 126, 56062.

[70] Christensen, L.; Nielsen, J. H.; Brandt, C. B.; Madsen, C. B.; Madsen, L. B.; Slater, C. S.; Lauer, A.; Brouard, M.; Johansson, M. P.; Shepperson, B. et al. Dynamic Stark control of torsional motion by a pair of laser pulses. Phys. Rev. Lett. 2014, 113, 073005.

[71] Normand, D.; Lompré, L. A.; ; Cornaggia, C. J. Phys. B 1992, 25, L497.

[72] Friedrich, B.; Herschbach, D. Phys. Rev. Lett. 1995, 74, 4623.

[73] Holmegaard, L.; Nielsen, J. H.; Nevo, I.; Stapelfeldt, H.; Filsinger, F.; Küpper, J.; Meijer, G. Phys. Rev. Lett. 2009, 102, 023001.

[74] Filsinger, F.; Küpper, J.; Meijer, G.; Holmegaard, L.; Nielsen, J. H.; Nevo, I.; Hansen, J. L.; Stapelfeldt, H. J. Chem. Phys. 2009, 131, 064309. 
[75] Nevo, I.; Holmegaard, L.; Nielsen, J. H.; Hansen, J. L.; Stapelfelt, H.; Filsinger, F.; Meijer, G.; Küpper, J. Phys. Chem. Chem. Phys. 2009, 11, 9912.

[76] Stapelfeldt, H.; Seideman, T. Rev. Mod. Phys. 2003, 75, 543.

[77] Vallance, C. Generation, characterisation, and applications of atomic and molecular alignment and orientation. Phys. Chem. Chem. Phys. 2011, 13, 14427 .

[78] Madsen, C. B.; Madsen, L. B.; Viftrup, S. S.; Johansson, M. P.; Poulsen, T. B.; Holmegaard, L.; Kumarappan, V.; Jørgensen, K. A.; Stapelfeldt, H. Manipulating the torsion of molecules by strong laser pulses. Phys. Rev. Lett. 2009, 102, 073007.

[79] Madsen, C. B.; Madsen, L. B.; Viftrup, S. S.; Johansson, M. P.; Poulsen, T. B.; Holmegaard, L.; Kumarappan, V.; Jørgensen, K. A.; Stapelfeldt, H. A combined experimental and theoretical study on realizing and using laser controlled torsion of molecules. J. Chem. Phys. 2009, $130,234310$.

[80] Hansen, J. L.; Nielsen, J. H.; Madsen, C. B.; Lindhardt, A. T.; Johansson, M. P.; Skrydstrup, T.; Madsen, L. B.; Stapelfeldt, H. Control and femtosecond time-resolved imaging of torsion in a chiral molecule. J. Chem. Phys. 2012, 136, 204310.

[81] Christiansen, L.; Nielsen, J. H.; Pentlehner, D.; Underwood, J. G.; Stapelfeldt, H. Alignment enhancement of molecules embedded in helium nanodroplets by multiple laser pulses. Phys. Rev. A 2015, 92, 053415 .

[82] Chatterley, A. S.; Shepperson, B.; Stapelfeldt, H. Three-dimensional molecular alignment inside helium nanodroplets. Phys. Rev. Lett. 2017, 119, 073202 .

[83] Pickering, J. D.; Shepperson, B.; Hübschmann, B. A. K.; Thorning, F.; Stapelfeldt, H. Alignment and imaging of the $\mathrm{CS}_{2}$ dimer inside helium nanodroplets. Phys. Rev. Lett. 2018, 120, 113202.

[84] Schouder, C.; Chatterley, A. S.; Calvo, F.; Christiansen, L.; Stapelfeldt, H. Structure determination of the tetracene dimer in helium nanodroplets using femtosecond strong-field ionization. Struct. Dyn. 2019, 6, 044301. 
[85] Pickering, J. D.; Shepperson, B.; Christiansen, L.; Stapelfeldt, H. Femtosecond laser induced Coulomb explosion imaging of aligned OCS oligomers inside helium nanodroplets. J. Chem. Phys. 2018, 149, 154306.

[86] Helm, H.; Bjerre, N.; Dyer, M. J.; Huestis, D. L.; Saeed, M. Images of photoelectrons formed in intense laser fields. Phys. Rev. Lett. 1993, 70, 3221 .

[87] Bordas, C.; Paulig, F. Photoelectron imaging spectroscopy: principle and inversion method. Rev. Sci. Instrum. 1996, 67, 2257.

[88] C. Y. Ng, E. Advanced Series in Physical Chemistry: Volume 10, Photoionization and Photodetachment; World Scientific Press, 2000.

[89] Suzuki, T. Femtosecond time-resolved photoelectron imaging. Annu. Rev. Phys. Chem. 2006, 57, 555 .

[90] Mabbs, R.; Grumbling, E. R.; Pichugin, K.; Sanov, A. Photoelectron imaging: an experimental window into electronic structure. Chem. Soc. Rev. 2009, 38, 2169.

[91] Rading, L.; Lahl, J.; Maclot, S.; Campi, F.; Coudert-Alteirac, H.; Oostenrijk, B.; Peschel, J.; Wikmark, H.; Rudawski, P.; Gisselbrecht, M. et al. A versatile velocity map ion-electron covariance imaging spectrometer for high-intensity XUV experiments. Appl. Sci. 2018, 8, 998.

[92] Lehmann, C. S.; Ram, N. B.; Janssen, M. H. M. Velocity map photoelectron-photoion coincidence imaging on a single detector. Rev. Sci. Instrum. 2012, 83, 093103.

[93] Zhao, A.; Sándor, P.; Weinacht, T. Coincidence velocity map imaging using a single detector. J. Chem. Phys. 2017, 147, 013922.

\section{Author biographies}

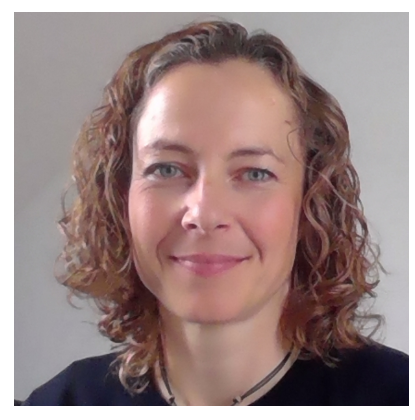

Claire Vallance is a Professor of Physical Chemistry at the University of Oxford, Tutorial Fellow in Physical Chemistry at Hertford College, and current President of the Faraday Division of the Royal Society of Chemistry. 
Her current research interests include chemical reaction dynamics and velocity-map imaging, microcavity spectroscopy, and applications of spectroscopy and mass spectrometry in medicine. She has published textbooks on reaction dynamics, astrochemistry, chemical kinetics, properties of gases, and symmetry and group theory in chemistry, and is a co-founder of the spin-out company Oxford HighQ.

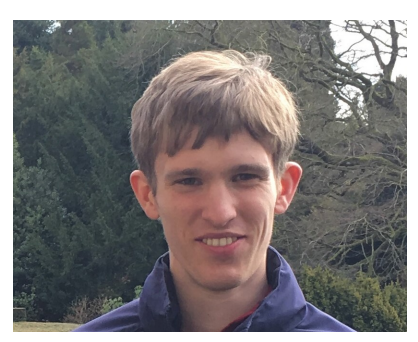

David Heathcote recieved an M.Chem. from the University of Oxford in 2016, going on to to study for a D.Phil. in Physical and Theoretical Chemistry in the research group of Claire Vallance. He has recently begun a post as a postdoctoral research associate in molecular scattering in the same research group. His research currently focusses on the scattering of electrons from molecules of astrophysical and astrochemical relevance.

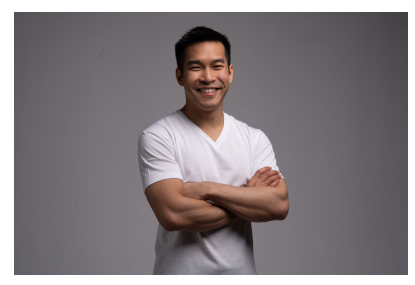

Jason Lee is a physical chemist in the Photon Science - Spectroscopy of Molecular Processes (FS-SMP) group at the Deutsches Elektronen-Synchrotron (DESY), Hamburg. He graduated from the University of Cambridge with a Bachelor's degree and Masters in Natural Sciences (Physical) in 2010, followed by a D.Phil. in Physical and Theoretical Chemistry from the University of Oxford in 2014. He continued at the University of Oxford as a postdoc before joining DESY in 2019. His research focusses on the development and application of novel imaging methods and technology to explore gas phase molecular dynamics." 